\title{
Vitamins D2 and D3 have overlapping but different effects on human gene expression revealed through analysis of blood transcriptomes in a randomised double-blind placebo-controlled food-fortification trial
}

\section{Louise Durrant}

University of Surrey

\section{Giselda Bucca}

University of Brighton https://orcid.org/0000-0001-5286-7771

\section{Andrew Hesketh}

University of Brighton https://orcid.org/0000-0001-5220-8733

Carla Moller-Levet

University of Surrey

\section{Laura Tripkovic}

University of Surrey

Huihai Wu

University of Surrey

Kathryn Hart

University of Surrey https://orcid.org/0000-0001-7494-9866

John Mathers

Newcastle University

Ruan Elliott

University of Surrey

\section{Susan Lanham-New}

University of Surrey

Colin Smith ( $\nabla$ c.smith6@brighton.ac.uk)

University of Brighton https://orcid.org/0000-0001-9042-2263

\section{Article}

Keywords: Vitamin D3, vitamin D2, 25-hydroxyvitamin D, human transcriptome, immunity, ethnic differences, Covid-19 
Posted Date: March 29th, 2021

DOI: https://doi.org/10.21203/rs.3.rs-354560/v1

License: (c) (i) This work is licensed under a Creative Commons Attribution 4.0 International License. Read Full License 
1 Vitamins $D_{2}$ and $D_{3}$ have overlapping but different effects on human gene expression revealed through analysis of blood transcriptomes in a randomised double-blind placebo-controlled food-fortification trial

5 Louise R. Durrant ${ }^{1,2}$, Giselda Bucca ${ }^{a, b, 1,3}$, Andrew Hesketh ${ }^{\mathrm{b}, 1}$, Carla Möller-Levet ${ }^{\mathrm{a}}$, 6 Laura Tripkovic $^{a}$, Huihai Wua ${ }^{a}$, Kathryn H. Harta, John C. Mathers ${ }^{c}$, Ruan M. Elliotta,

7 Susan A. Lanham-Newa \& Colin P. Smitha,b, 3*

a Department of Nutritional Sciences, School of Biosciences and Medicine, Faculty of Health and Medical Sciences, University of Surrey, Guildford, UK

${ }^{\mathrm{b}}$ School of Pharmacy and Biomolecular Sciences, University of Brighton, Brighton,

12 UK

${ }^{\mathrm{c}}$ Human Nutrition Research Centre, Population Health Sciences Institute, Newcastle

14 University, UK

1 These authors contributed equally

${ }^{2}$ Current address: Yakult UK Ltd., Anteros, Odyssey Business Park, West End

Road, South Ruislip, HA4 6QQ; formerly known as Louise Wilson.

${ }^{3}$ Current address: School of Pharmacy and Biomolecular Sciences, University of

20 Brighton, Brighton, UK

*For correspondence: Prof Colin P. Smith, School of Pharmacy and Biomolecular

Sciences, University of Brighton, Huxley Building, Moulsecoomb, Brighton, BN2 4GJ,

UK; Tel. +44 (0)1273 642048. Email: genomics@cpsmith.net

\section{Uploaded to preprint server:}

medRxiv 2020.12.16.20247700; doi: https://doi.org/10.1101/2020.12.16.20247700

Made available under a CC-BY 4.0 International license.

Key words: Vitamin $D_{3}$, vitamin $D_{2}$, 25-hydroxyvitamin $D$, human transcriptome, immunity, ethnic differences, Covid-19

\section{Abstract}

33 For the first time we report the relative influences of vitamin $D_{2}$ and vitamin $D_{3}$ on 34 genome-wide gene expression in whole blood from healthy women, representing two 
ethnic groups, white European and South Asian. In this randomised placebocontrolled trial, participants were given daily physiological doses (15 $\mu \mathrm{g})$ of either vitamin $D_{2}$ or $D_{3}$ for 12 weeks and changes in the transcriptome were compared relative to the transcriptome at baseline. While there was some overlap in the repertoire of differentially expressed genes after supplementation with each vitamin $D$ source, most changes were specific to either vitamin $D_{3}$ or vitamin $D_{2}$, suggesting that each form of the vitamin may have different effects on human physiology. Notably, following vitamin $D_{3}$ supplementation, the majority of changes in gene expression reflected a down-regulation in the activity of genes, many encoding components of the innate and adaptive immune systems. These are consistent with the emerging concept that vitamin D orchestrates a shift in the immune system towards a more tolerogenic status. Moreover, gene expression associated with type 1 and type 2 interferon activity differed following supplementation with either vitamin $D_{2}$ or vitamin $D_{3}$, with only vitamin $D_{3}$ having a stimulatory effect. Interferons play a critical role in the innate response to infection and aberrant type 1 interferon signalling has recently been implicated in severe Covid-19 disease. The observed differences in gene expression after supplementation with vitamin $D_{2}$ compared with vitamin $D_{3}$ warrant a more intensive investigation of the biological effects of the two forms of vitamin D on human physiology..

\section{Author Contributions}

CPS and SL-N conceived of the study. CPS, GB and SL-N supervised the project. analyzed and interpreted the transcriptome data, CPS, AH and GB wrote the first draft of the manuscript, $\mathrm{AH}$ generated the figures and all authors contributed to the

60 final manuscript.

\section{Significance statements}

This study suggests that the influence of vitamins $D_{2}$ and $D_{3}$ on human physiology may not be the same, as deduced from differences in gene expression within whole blood. 
South Asian participants were found to respond differently to vitamin D

supplementation at the transcriptome level from white Europeans.

The differentially expressed immune pathways identified in this study are consistent with vitamin $\mathrm{D}$ orchestrating a more tolerogenic immune status and this could be relevant in the context of the severity of immune response to viral infections such as Covid-19.

The potential relevance of this study to severe Covid-19 disease is highlighted by our observed enhancement of type 1 interferon signalling by vitamin $D_{3}$, but not vitamin $D_{2}$.

\section{Introduction}

Vitamin D is a pro-hormone that is critical for good health. While vitamin D's function in maintaining the musculo-skeletal system is best understood, it fulfils myriad roles in human physiology, including maintaining a healthy immune system ${ }^{1,2}$. Vitamin $D$ deficiency (defined as serum concentrations of 25-hydroxyvitamin $D(25(O H) D)$ below $25 \mathrm{nmol} / \mathrm{L}$ ) or inadequacy (defined as serum level of 25(OH)D below 50 $\mathrm{nmol} / \mathrm{L}$ ) is considered a global pandemic and a public health issue of great importance in the human population, especially in older people, individuals not exposed to sufficient sunlight and, importantly, ethnic minority groups with dark skin tone ${ }^{3,4}$. Vitamin $\mathrm{D}$ deficiency is considered to have many detrimental effects on human health which include increased risk of osteoporotic and stress fractures, increased risk of developing cardiovascular diseases and some cancers, and poor modulation of the immune system ${ }^{5,6}$. Low vitamin $D$ status is associated with higher mortality, including death from cancer ${ }^{7}$.

Vitamin $D_{3}$ (cholecalciferol) is produced in the skin by the action of ultraviolet $B$ radiation from the sun. Following two successive hydroxylation steps, in the liver and kidney, respectively, the active form of the vitamin, 1,25-dihydroxyvitamin $D_{3}$ $\left(1,25(\mathrm{OH}) \mathrm{D}_{3}\right)$ binds to the intracellular vitamin $\mathrm{D}$ receptor (VDR) and, in complex with

100 the retinoid $X$ receptor $(R X R)$, the heterodimer regulates expression of hundreds of genes through the vitamin $\mathrm{D}$ response element (VDRE) ${ }^{8}$. Vitamin $\mathrm{D}$ can also be 
obtained from foods or as a supplement. Both the plant/fungus-derived vitamin $D_{2}$ (ergocalciferol) and the animal-derived $\mathrm{D}_{3}$ forms are available as nutritional supplements. Both can be hydroxylated into their active forms and bind to the VDR with similar affinity, but there are differences in their catabolism and in their binding affinity to vitamin $\mathrm{D}$ binding protein (DBP), the major vitamin $\mathrm{D}$ transport protein in blood. Vitamin $\mathrm{D}_{2}$ binds DBP with lower affinity and is catabolised faster ${ }^{9,10}$.

The functional equivalence, or otherwise, of vitamins $D_{2}$ and $D_{3}$ for human health has been a subject of much debate in recent years, with some authors suggesting that the two compounds have equal efficacy while others provide evidence that vitamin $D_{3}$ increases circulating serum $25(\mathrm{OH}) \mathrm{D}$ concentration more efficiently than vitamin $D_{2}{ }^{11-15}$. Current guidance given to the general public by the US National Institutes of Health (NIH), the UK Department of Health and Social Care, and other government bodies around the world lack acknowledgement that the two forms of vitamin D are not considered equivalent or equally effective when used at nutritional (physiological) doses, while at high doses vitamin $\mathrm{D}_{2}$ is less potent. The NIH state that most evidence indicates that vitamin $\mathrm{D}_{3}$ increases serum $25(\mathrm{OH}) \mathrm{D}$ levels to a greater extent and maintains these higher levels longer than vitamin $D_{2}$, even though both forms are well absorbed in the gut ${ }^{16}$.

Although vitamin $D$ is best known for its role in maintaining bone health and calcium homeostasis, it also exerts a broad range of extra-skeletal effects on cellular physiology and on the immune system ${ }^{17-20}$ and multiple studies have linked poor vitamin D status with the pathogenesis of immune mediated inflammatory diseases 21. Vitamin $D$ is essential for human health and it is recommended that individuals maintain serum concentrations of at least $25 \mathrm{nmol} / \mathrm{L}$ of $25(\mathrm{OH}) \mathrm{D}$ (the accepted biomarker of systemic vitamin D status), throughout the year and throughout the life course ${ }^{22}$. However, it can be difficult to sustain such levels of serum 25(OH)D yearround from diet and sunlight alone, particularly amongst 'at-risk' groups, such as those with limited sun exposure or those with darker skin tone living in northern

132 latitudes (above $\left.40^{\circ} \mathrm{N}\right)$.

134 In acute studies comparing the efficacy of vitamins $D_{2}$ and $D_{3}$ in raising serum $13525(\mathrm{OH}) \mathrm{D}$ concentration, vitamin $\mathrm{D}_{2}$ is less effective than $\mathrm{D}_{3}$ when given as single 
bolus ${ }^{11}$. However, the findings from studies following the daily administration of $D_{2}$

137 or $\mathrm{D}_{3}$ over longer time-periods are more equivocal, with clinical trials showing higher

138 efficacy of $\mathrm{D}_{3}{ }^{23,24}$ or equal efficacy ${ }^{25}$. In a recent meta-analysis, supplementation

139 with vitamins $D_{2}$ and $D_{3}$ were found equally effective in raising $25(\mathrm{OH}) \mathrm{D}$

140 concentrations in infants up to 1 year of age ${ }^{26}$. It should be noted that

141 supplementation with one form of vitamin D can reduce circulating concentrations of

142 the other form. For example, our recent comparative trial of $D_{2}$ versus $D_{3}$ revealed

143 that serum $25(\mathrm{OH}) \mathrm{D}_{3}$ concentration was reduced over the 12-week intervention in

144 participants given vitamin $\mathrm{D}_{2}$, compared with the average 12-week concentration in

145 participants given placebo $\left({ }^{24}\right.$, see Fig. $1 \mathrm{C}$, for example). The implications of such

146 reciprocal depletion remain to be explored.

148 There is limited understanding of the effects of vitamin D supplementation on gene

149 expression in vivo in humans because of the diversity of experimental designs used.

150 This includes: (i) diverse sampling intervals, ranging from hours to years following

151 vitamin D supplementation ${ }^{27,28}$; (ii) use of substantially different doses ranging from

152 physiological (moderate) to supra-physiological doses; and (iii) relatively small

153 numbers of participants were recruited so that most studies were considerably

154 underpowered ${ }^{28,29}$. Currently, there is no robust evidence, from in vivo human

155 genome-wide expression analysis, about which specific cellular pathways are

156 influenced by vitamin D supplementation. Moreover, the influence of vitamin $D_{2}$, as

157 distinct from vitamin $D_{3}$, on gene expression in humans has not yet been evaluated,

158 even though vitamin $D_{2}$ is used widely as a supplement and food fortificant.

160 We have addressed this gap in knowledge by investigating gene expression in a

161 relatively large cohort of healthy white European and South Asian women who

162 participated in a randomised double-blind placebo-controlled trial that compared the relative efficacy of vitamins $D_{2}$ and $D_{3}$ in raising serum $25(\mathrm{OH}) \mathrm{D}$ concentration. The study concluded that vitamin $D_{3}$ was superior to vitamin $D_{2}$ in raising serum $25(O H) D$ concentration ${ }^{24}$. As an integral part of the original study design, we also investigated gene expression in the participants over the 12-week trial period. This allowed us to examine the effects of vitamin D supplementation on the transcriptome and to determine whether these effects might differ depending on supplementation with

169 vitamin $D_{2}$ compared with vitamin $D_{3}$. We therefore sampled blood at the beginning, 
middle and end of the 12-week intervention and quantified changes in the whole blood transcriptome. Surprisingly, we observed that the two vitamins exerted overlapping but different effects on the human blood transcriptome. Our findings support the hypothesis that the biological effects of vitamin $D_{2}$ and $D_{3}$ may differ in humans and suggest that a more comprehensive analysis of the biological effects of

175 the two forms of vitamin D on human physiology is warranted. In this context, it is important to note that a recent meta-analysis of vitamin D supplementation trials found that reduced cancer mortality was seen only with vitamin $D_{3}$ supplementation, not with vitamin $D_{2}$ supplementation, and indicated that all-cause mortality was significantly lower in trials with vitamin $D_{3}$ supplementation than in trials with vitamin $\mathrm{D}_{2}{ }^{7}$.

\section{Results}

183 The study volunteers were South Asian (SA) and white European (WE) women based in the United Kingdom, aged between 20 and 64 years $\left(n=335 ;{ }^{24}\right)$ and participation was for 12 weeks over the winter months (October to March, in Surrey, UK; latitude, $\left.51^{\circ} 14^{\prime} \mathrm{N}\right)$. Serum measurements, including concentrations of total $25(\mathrm{OH}) \mathrm{D}, 25(\mathrm{OH}) \mathrm{D}_{2}$ and $25(\mathrm{OH}) \mathrm{D}_{3}$, were determined from fasting blood samples taken at the start (baseline defined as Visit 1 (V1)) and at weeks 6 (V2) and 12 (V3). To determine whether changes in serum 25(OH)D concentration as a result of vitamin D supplementation are associated with physiological changes at the level of gene expression, we investigated the whole blood transcriptome from a representative subset of the study participants $(n=98)$ using total RNA isolated from V1 and V3 blood samples and Agilent Human Whole Genome 8× 60K v2 DNA microarrays (Fig. 1). Participants for transcriptome analysis were selected to provide comparable numbers between the placebo and the two vitamin D treatment groups, covering the full range of serum responses to supplementation within the $D_{2}$ and $D_{3}$ treatment groups, as judged from the measured changes in serum $25(\mathrm{OH}) \mathrm{D}_{2}$ or $25(\mathrm{OH}) \mathrm{D}_{3}$ concentration between V1 and V3 (Supplementary Data File 1). 
202 Following microarray data quality control, transcriptome data were available for both

203 the V1 and V3 samples of 97 study participants (Fig. 1b); the RNA from one

204 participant failed quality control and was excluded. Similar to data reported

205 previously for the entire study cohort ${ }^{24}$, the sub-set of participants in the present

206 study showed increased concentrations of $25(\mathrm{OH}) \mathrm{D}_{2}$ and $25(\mathrm{OH}) \mathrm{D}_{3}$ only after

207 supplementation with vitamin $D_{2}$ and vitamin $D_{3}$, respectively, and higher total

208 25(OH)D in both treatment groups (Fig. 1c). In the vitamin $\mathrm{D}_{3}$ treatment groups the

209 mean $25(\mathrm{OH}) \mathrm{D}_{3}$ serum levels rose by $59 \%$ (WE) and $116 \%$ (SA) over the 12-week

210 intervention. Conversely, mean serum $25(\mathrm{OH}) \mathrm{D}_{3}$ concentrations fell across the 12

211 weeks of the study in the placebo groups who did not receive additional vitamin D;

$21225(\mathrm{OH}) \mathrm{D}_{3}$ baseline vitamin $\mathrm{D}$ levels in the SA ethnic group tended to be lower than

213 for the WE group and dropped, respectively, by $23 \%$ and $29 \%$ relative to baseline $\mathrm{V} 1$

214 (Fig. 1c; Supplementary Data File 1). It is notable that, in the vitamin $\mathrm{D}_{2}$ treatment

215 group, serum $25(\mathrm{OH}) \mathrm{D}_{3}$ concentration decreased to a greater extent over the 12-

216 week period, by $52 \%$ (SA) and $53 \%$ (WE); the implications of this are considered

217 further in the Discussion. Serum $25(\mathrm{OH}) \mathrm{D}_{2}$ was low in the absence of specific

218 supplementation, typically less than $5 \mathrm{nmol} / \mathrm{L}$ (152/162 samples analysed). Serum

219 calcium concentration, appropriately adjusted for serum albumin concentration, was

220 maintained within a normal clinical range across all sample groups, while the

221 concentration of parathyroid hormone (PTH) was stable between V1 and V3 only

222 within the SA placebo group, the WE vitamin $D_{2}$ and WE vitamin $D_{3}$ intervention

223 groups. PTH concentrations showed an increase at V3 compared with V1 in the WE

224 placebo group, but a decrease in both the vitamin $D_{2}$ and $D_{3}$ intervention groups in

225 the SA ethnic cohort.

226

Effects of supplementation with either vitamin $D_{2}$ or $D_{3}$ on global gene expression

230 Filtering of the normalised microarray data to select for probes showing signals at

231 least $10 \%$ above background in at least $41(\sim 20 \%)$ of the arrays yielded transcript

232 abundance data from 20,662 probes corresponding to 17,588 different genomic

233 features (12,436 of which were annotated with an ENTREZ gene identifier). Using

234 the filtered data, we identified significant differences in the transcriptional responses

235 occurring across the 12-week V1 to V3 period of the study between the vitamin $\mathrm{D}_{2}$, 
vitamin $D_{3}$ and placebo treatment groups for each ethnic cohort and, in a separate analysis, to determine significant changes within each group between the V3 and V1 sampling points (Fig. 2; Supplementary Data File 2). The former was tested in a 'difference-in-difference' analysis according to the generalised null hypothesis [treatment1.V3 - treatment1.V1] - [treatment2.V3 - treatment2.V1] $=0$ (Fig. 2a, rows 7-12), and the latter using treatment.V3 - treatment.V1 = 0 (Fig. 2a, rows 1-6).

No significant changes in probe signals (adj.P.Val<=0.05) were identified in the 'difference-in-difference' analysis for the data for the WE ethnic group, but five were found in the corresponding analysis of the SA cohort (Fig. 2a). Furthermore, difference-in-difference analysis of the entire study data, ignoring ethnicity, did not yield statistically significant changes between the $D_{2}$ or $D_{3}$ treatment groups and the placebo control (data not shown). We note that another 'difference-in-difference' study on the influence of vitamin D on gene expression (the BEST-D trial) failed to identify any significant changes in gene expression following long-term vitamin $D$ treatment in a Caucasian cohort ${ }^{27}$. The observed $\log _{2}$ fold-change $\left(\log _{2} F C\right)$ in signal abundances between V3 and V1 in the study typically fall well within $+/-1$, and in such a context it is likely that the sample sizes used within the study restrict our ability to reliably identify individual gene expression responses using this highly conservative approach. We consider that inter-individual differences in human populations are likely to hinder detection of small statistically significant gene expression changes across treatment groups from microarray-derived data; however, statistically significant differences are detected when evaluating gene expression changes within an individual across time (as described below where we examine paired observations with two time points per subject). The five significant changes that were identified as occurring in response to treatment of the SA ethnic group with vitamin $D_{3}$, relative to the placebo, are summarised in Supplementary Fig. 1. These are driven primarily by the marked changes in the placebo group between the V1 and V3 sampling points, and may be associated with the sustained low serum $25(\mathrm{OH}) \mathrm{D}_{3}$, or high $\mathrm{PTH}$, concentrations observed in this group of subjects. One of these differentially expressed genes encodes the CAMP response element binding protein CREB1 (Fig. 2b) which is part of the cAMP-PKA-CREB signaling pathway in bone cells which contributes to the regulation of skeletal metabolism in response to $\mathrm{PTH}$ concentrations ${ }^{30}$. 
271 In contrast to the difference-in-difference analysis, the direct within-group

272 comparisons of transcript abundance measurements at V3 versus V1 per subject

273 identified large numbers of significant changes in gene expression, most notably in

274 the WE $D_{2}, W E D_{3}$ and WE placebo groups (Fig. 2a and c, Supplementary Data

275 File 2). These significant gene expression changes in the WE ethnic cohort arising in

276 the vitamin $\mathrm{D}$ treatment groups are considered in more detail below. While there is

277 some overlap in the groups of differentially expressed genes, only $13 \%$ of down-

278 regulated differentially expressed genes (102 of 774) were common between the two

279 vitamin D treatment groups, while $28 \%$ (216 of 774 ) and 59\% (456 of 774 ) were

280 uniquely down-regulated by $\mathrm{D}_{2}$ and $\mathrm{D}_{3}$, respectively (excluding those additionally

281 down-regulated in the placebo group over the equivalent 12-week intervention)(Fig

282 2c). As previously noted above, the serum $25(\mathrm{OH}) \mathrm{D}_{3}$ concentration fell over the $12-$

283 week intervention period in the vitamin $\mathrm{D}_{2}$-treated group; thus, changes in gene

284 expression observed in this group could a priori either be due to the influence of

285 vitamin $D_{2}$ itself, or could be attributable to the depletion of the endogenous

$28625(\mathrm{OH}) \mathrm{D}_{3}$ reserves. It was therefore important to have included comparative

287 transcriptomic analysis of the placebo group in this study to distinguish gene

288 expression changes resulting from vitamin $\mathrm{D}_{2}$ per se from those changes arising due

289 to the depletion of $25(\mathrm{OH}) \mathrm{D}_{3}$ levels in this vitamin $\mathrm{D}_{2}$ treatment group.

291 It is notable that expression of a large number of genes was altered between the first

292 and last visits (V1 to V3) in the non-treated placebo group. While some of these

293 changes will be attributable to the effects of the vitamin D depletion observed in this

294 group over the course of the study, there is significant over-representation of genes

295 known to exhibit seasonal differences in gene expression ${ }^{31}$ (Supplementary Fig.

296 2). There is however no unique association of seasonally expressed genes with the

297 data for the WE placebo group; a similar significant enrichment is also observed for

298 the significant changes in gene expression identified in the WE $D_{2}$ and WE $D_{3}$

299 treatment groups (Supplementary Fig. 2) and therefore it is unlikely that this

300 apparent seasonal effect exclusively reflects vitamin D-specific effects. There was no

301 overlap between genes significantly down-regulated exclusively in the placebo group

302 and those significantly up-regulated exclusively in the vitamin D treatment groups (or

303 vice versa). Consistent with the 12-week period of the study, seasonal changes in 
gene expression are therefore a background feature of all the data collected, and is therefore a factor that needs to be considered when undertaking such time course studies.

In the group of probe signals with significantly reduced abundance in the V3 samples compared with $\mathrm{V} 1$ in the data for the vitamin $\mathrm{D}_{2}$ and $\mathrm{D}_{3}$ supplemented participants, but not in the placebo participants, Probe A_33_P3275751, detecting SEC14L1 gene transcription showed the largest decrease in response to both forms of vitamin $D$

312 (Fig. 2d). This probe was originally designed to detect 'transcript variant 7' and

313 hybridizes to a location at the 3' end of the SEC14L1 locus, detecting several splice

314 variants. High expression of SEC14L1 is significantly associated with

315 lymphovascular invasion in breast cancer patients, where transcript abundance

316 correlates positively with higher grade lymph node metastasis, and poor prognostic

317 outcome ${ }^{32}$. Its overexpression is also frequent in prostate cancer where SEC14L1

318 has been identified as a potential biomarker of aggressive progression of the disease $31933,34$.

Genes significantly down-regulated by both $D_{2}$ and $D_{3}$, but not placebo, are enriched for functions associated with immune responses

Given the relatively small sample sizes used in this study it is considered more appropriate to examine enrichment of cellular pathways among differentially expressed genes rather than focusing on changes in individual genes. Functional enrichment analysis of the genes represented by the probes specifically repressed by vitamins $D_{2}$ and $D_{3}$ (blue data points in Fig. $2 d$ ) suggests that both supplements have suppressive effects on the immune response in the WE ethnic group (Fig. 3 and Supplementary Data File 3). Indeed, a significant ( $p=1.98 E-06)$ proteinprotein interaction network derived from the Homo sapiens medium confidence interactions curated in the STRING database ${ }^{35}$ is centred on a highly connected group of proteins associated with the innate immune response, neutrophil degranulation and leukocyte activation (Fig. 3a; all protein groups are detailed in Supplementary Data File 3). This includes the histone acetyltransferase, EP300,

336 known to function as a transcriptional coactivator with VDR, the vitamin D receptor 337 protein ${ }^{36}$. Collectively, these findings are consistent with the emerging view that 
338 vitamin $\mathrm{D}$ exposure leads to a shift from a pro-inflammatory to a more tolerogenic

339 immune status ${ }^{1,2}$.

341 Genes significantly up-regulated by both $D_{2}$ and $D_{3}$, but not placebo, include 342 components of histone H4 and the spliceosome

344 A similar analysis of the probes specifically induced by $D_{2}$ and $D_{3}$ (red points in Fig.

$3452 \mathrm{~d})$ produced a significant $(p=4.99 \mathrm{E}-09)$ protein-protein interaction network

346 enriched primarily for mitochondrial and ribosomal proteins, but also involving two

347 subunits of histone H4 and SNRPD2, a core component of the SMN-Sm complex

348 that mediates spliceosomal snRNP assembly (Fig. 3b).

Comparative functional enrichment analysis supports roles for vitamins $D_{2}$ and $D_{3}$ in the suppression of immunity, and in chromatin modification and spliceosome function

As a complementary approach to the functional enrichment analysis of specific subsets of genes whose expression is altered by supplementation with vitamin $D_{2}$ and $D_{3}$ but not by placebo treatment, a comparative functional enrichment analysis of all significant changes in each treatment group was performed (see Methods).

358 This aimed to identify functional categories that are more extensively affected by vitamin $D_{2}$, or by vitamin $D_{3}$, or by both vitamins $D_{2}$ and $D_{3}$, than by the placebo, and took into consideration the changes occurring in the placebo treatment group across the 12 weeks of the study (Figs. 4 and 5; Supplementary Data File 4). Consistent with the earlier analysis, functional categories associated with immunity and immune response pathways are prominent among those genes repressed by vitamin $D$ supplementation (Fig. 4), while mitochondrial, ribosomal and spliceosomal functions are prominent in the induced genes (Fig. 5). Although the two vitamin treatments share many common categories identified from this analysis, the results also highlight some differences between the respective effects of $D_{2}$ or $D_{3}$ supplementation. For example, 'histone exchange' is significant only in the vitamin $D_{2}$ up-regulated genes, and 'chromatin modifying enzymes' are significant only in the $D_{3}$ down-regulated genes. 
372 Overall, the observed differences in gene expression from the blood transcriptome

373 presented in this study suggest that the physiological effects of vitamin $D_{3}$ and $D_{2}$

374 may be dissimilar.

Weighted gene correlation network analysis (WGCNA) identifies modules of co-expressed genes that significantly correlate with serum markers of vitamin D supplementation in the WE and SA ethnic groups

WGCNA quantifies both the correlations between individual pairs of genes or probes across a data set, and also the extent to which these probes share the same neighbours ${ }^{37}$. The WGCNA process creates a dendrogram that clusters similarly abundant probes into discrete branches, and subsequent cutting of the dendrogram yields separate co-expression modules, representing putatively co-regulated sets of genes. The first principal component of the expression matrix of each module defines the expression profile of the eigengene for the module, and this can then be correlated with experimental metadata. By allowing phenotypic traits to be associated with relatively small numbers (tens) of module eigengenes, instead of thousands of individual variables (i.e. gene probes), WGCNA both alleviates the multiple testing problem associated with standard differential expression analysis and also directly relates experimental traits to gene expression data in an unsupervised way that is agnostic of the experimental design.

WGCNA was used to construct separate signed co-expression networks for the WE and SA ethnic groups as described in the Methods, and Pearson correlations between expression of the module eigengenes and serum $25(\mathrm{OH}) \mathrm{D}_{2}, 25(\mathrm{OH}) \mathrm{D}_{3}$, total 25(OH)D and parathyroid hormone (PTH) concentrations were calculated (Fig.

3986 and Supplementary data files 6 - 8). These results therefore ignore whether the data originates from the vitamins $D_{2}, D_{3}$ or placebo treatment groups and focus solely on the relationship between the serum metabolite concentrations and gene expression. A significant negative correlation was observed between $25(\mathrm{OH}) \mathrm{D}_{2}$ concentration and expression modules in the WE co-expression network that are enriched for immune-associated functions (midnight blue and pink modules in Fig.

404 6a). This is consistent with the results from the different analytical approaches 405 (presented in Figs 3 and 4). Similarly, the only module exhibiting a significant 
positive correlation with $25(\mathrm{OH}) \mathrm{D}_{2}$ (and total $25(\mathrm{OH}) \mathrm{D}$ concentration), the red expression module, is enriched for GO categories associated with the ribosome and mRNA processing (Supplementary Data File 6). No significant correlations with serum $25(\mathrm{OH}) \mathrm{D}_{3}$ concentrations were detected in the WE cohort.

Interestingly, stronger and more numerous correlations were observed in the coexpression network for the SA cohort (Fig. 6b). In agreement with the WE network, an expression module significantly associated with the ribosome (black module in Fig. 6b) showed a general positive correlation with total $25(\mathrm{OH}) \mathrm{D}$ concentration whereas a module enriched for histone binding (turquoise) had a significant negative correlation with $25(\mathrm{OH}) \mathrm{D}$ concentration. Strikingly however, modules enriched for immune response functions (midnightblue, orange, purple, yellow in Fig. 6b) were consistently positively correlated with total $25(\mathrm{OH}) \mathrm{D}$ and usually $25(\mathrm{OH}) \mathrm{D}_{3}$, but not $25(\mathrm{OH}) \mathrm{D}_{2}$ concentrations in the SA network, and negatively correlated with PTH concentrations. This raises the possibility that vitamin $D$ supplementation may exert different effects on the immune system depending on ethnicity of the individual, may indicate that PTH status has an influence on the outcome and may reflect physiological differences resulting from the low baseline vitamin D status in SA women. In this context we observed that PTH concentrations at the V1 sampling point in the SA cohort were elevated compared with those in the WE cohort (Fig. 1c).

\section{Gene sets that respond to interferon alpha and gamma show divergent} behaviour in the vitamin $D_{2}$ and $D_{3}$ treatment groups of the WE cohort

The molecular signatures database (MSigDB) hallmark gene sets represent coherent gene expression signatures related to well-defined biological states or processes (https://www.gsea-msigdb.org/gsea/msigdb/). Gene set enrichment analysis (GSEA) was performed to identify statistically significant (padjust<=0.05), concordant changes in expression of these hallmark sets between the V3 and V1 sampling times for the placebo, vitamin $D_{2}$, and vitamin $D_{3}$ treatment groups (Fig. 7 and Supplementary Data File 9). Interestingly, in the WE cohort, the gene sets defining the signature responses to interferon alpha and gamma exhibited divergent behaviour in the vitamin $D_{2}$ and $D_{3}$ treatment groups. This is evident as significant up-regulation over the course of the intervention study in the $D_{3}$ group but significant 
440 down-regulation in the $\mathrm{D}_{2}$ group. This differed from the SA cohort where significant

441 down-regulation was observed in the $D_{3}$ group.

\section{Discussion}

445 Vitamin D intake down-regulates expression of many genes including those encoding multiple pathways involved in innate and adaptive immunity

448 In the present study we shed light on the physiological effects of the two forms of

449 vitamin D commonly used as food-fortificants or vitamin supplements. Using data

450 from the same intervention study, previously we reported that vitamin $D_{3}$

451 supplementation is more effective than supplementation with vitamin $D_{2}$ in raising

452 serum 25(OH)D concentration ${ }^{24}$. However, other studies have reported that the two

453 forms of vitamin D are equally effective in raising serum $25(\mathrm{OH}) \mathrm{D}$ concentration ${ }^{25,26}$.

454 In the present study we examined gene expression changes in vivo following

455 supplementation with either vitamin $D_{2}$ or $D_{3}$ (or placebo) in healthy women from the

456 South East of the UK. Here we extend the results reported by Tripkovic et al. (2017)

457 by conducting whole genome transcriptome analysis in 97 of the original cohort of

458335 women from two ethnic backgrounds, South Asian (SA) and white European

459 (WE). We characterised gene expression changes in the human blood transcriptome

460 in response to vitamin $D_{2}$ or $D_{3}$ supplementation over a 12-week treatment

461 intervention over the winter months; the randomised groups were provided with

462 physiological $(15 \mu \mathrm{g})$ daily doses of vitamin $\mathrm{D}_{2}$ or vitamin $\mathrm{D}_{3}$ fortified foods, or

463 identical foods without added vitamin D (placebo). We found extensive changes in

464 gene expression in all three treatment groups, some of which were unique to the

465 vitamin $\mathrm{D}_{2}$-treated or $\mathrm{D}_{3}$-treated groups, with the majority exhibiting downregulation

466 of transcription over the 12-week intervention period. Our success in detecting

467 statistically significant differential gene expression in this study stems partly from the

468 longitudinal design where it was possible to evaluate statistical differences in

469 transcript levels within each group (from baseline to 12-week measurement). This

470 approach partly circumvents the problems with inter-individual differences in human

471 studies which make it difficult to detect robust changes between different groups of

472 individuals. Furthermore, the statistical analysis approach examined pairwise 
differences in gene expression from the V1 and V3 samples, which helps to reduce the impact of inter-individual differences on statistical significance.

This in vivo human transcriptome study has demonstrated long-term effects on gene expression. The majority of differentially expressed genes identified in this study were suppressed by vitamin D supplementation, and many of these encoded pathways were involved in immunity. Many of these observed gene expression changes are consistent with vitamin D exerting a modulating effect on the immune system, leading towards a more tolerogenic state, a concept reviewed by ${ }^{1}$. The findings from this study are based on an analysis of 97 individuals divided equally between the three treatment groups. Our ability to detect differences in the effects of vitamin $D_{2}$ and vitamin $D_{3}$ may have been negatively impacted by the inclusion of two different ethnic groups among the 97 participants, since the transcriptome results from the two ethnic groups are clearly different. It will be very important to independently replicate this study using a much larger cohort in order to verify, or otherwise, the key findings from this study. From power calculations it is considered that for a whole human microarray-based gene expression study of this nature, and with gene expression changes of the magnitude we observe, at least 400 participants of each ethnic group should be recruited for each treatment, giving a total cohort size of 2,400. The biological interpretation of our findings in the present study should be considered as preliminary in this context, requiring independent verification.

It is difficult to compare the findings of the present study with other reported in vivo studies because of the considerable differences in experimental design, including the use of supra-physiological vitamin D doses (up to 2,000 $\mu \mathrm{g}$ single bolus doses), different population types and small sample sizes which make statistical analysis not feasible (e.g. ${ }^{28,29}$ ). Furthermore, our study is unique in that it compared gene expression in participants given either of the two commonly used forms of vitamin $D$, vitamin $D_{2}$ and vitamin $D_{3}$. Intriguing, this revealed that there were considerable differences in the gene expression profiles following each nutritional treatment. For

504 example, excluding genes that were also differentially expressed over the 12-week intervention in the placebo group, only $13 \%$ of down-regulated differentially 
expressed genes were common between the two treatment groups while $28 \%$ and $59 \%$ were uniquely down-regulated by vitamins $D_{2}$ and $D_{3}$, respectively (Figure $2 c$ ).

It is questionable whether it is useful to extrapolate the results from single high dose bolus and in vitro studies such as those described by ${ }^{38}$ with this sustained dietary intake in vivo study. In the former study 15 'vitamin D-responsive' genes were identified as important mediators of innate and adaptive immunity. Five of these 15 genes are also identified as differentially expressed in our present study (CD93, CEBPB, THBD, THEMIS2 and TREM1). However, in the present study these five genes were down-regulated by vitamin D intake (Supplementary Data File 2) whereas they are reported to be up-regulated in the studies reported by ${ }^{38}$. These contrasting results highlight the difficulties with comparing data from different experimental designs. As argued previously, because of the relatively small sample sizes investigated and the small effect sizes, this study has focussed on identification of statistically significant pathway enrichment following sustained vitamin $D$ supplementation, rather than a gene-focussed analysis. Nevertheless, we consider it useful to consider the respective roles of these five specific gene products since their down-regulation by vitamin $\mathrm{D}$ would make sense from a biological perspective. In our study the gene encoding thymocyte selection-associated family member 2 (THEMIS2) is down-regulated after vitamin $\mathrm{D}_{2}$ supplementation, the CD93-encoding gene is down-regulated following $D_{2}$ and $D_{3}$ supplementation, while the genes encoding thrombomodulin (THBD) and the CCAAT enhancer-binding protein beta $(C E B P B)$ are down-regulated following $D_{3}$ supplementation. THBD is a key vitamin $D$ target in peripheral blood mononuclear cells (PBMCs) with the function of reducing blood clots while at the same time preventing the pro-inflammatory consequences of NF-kB signalling. The THBD and CD93 genes are next to each other, share the same super-enhancer, and their transcription start site regions respond to $1,25(\mathrm{OH})_{2}$ $D_{3}$. $C E B P B$ encodes a transcription factor important in the regulation of genes involved in immune and inflammatory responses. TREM1 encodes triggering receptor expressed on myeloid cells which stimulates neutrophil and monocytemediated inflammatory responses. It triggers release of pro-inflammatory chemokines and cytokines, as well as increased surface expression of cell activation markers. TREM-1 amplifies inflammatory responses that are triggered by bacterial and fungal infections and is a crucial mediator of septic shock ${ }^{39}$. 
Another cellular function that is affected by vitamin $D_{2}$ and $D_{3}$ supplementation is chromatin modification and remodelling. The EP300 gene is down-regulated by both vitamins, but unaffected in the placebo group. EP300 encodes a histone acetylase that regulates transcription by chromatin remodelling, in particular histone acetylation of $\mathrm{H} 3$ at lysine-27, representing an epigenetic modification which activates transcription ${ }^{40}$. Importantly, EP300 is also a coactivator of VDR.

547 Functional categories of genes enriched among the upregulated genes, following 548 supplementation with either vitamin $\mathrm{D}_{2}$ or $\mathrm{D}_{3}$, include translation, mitochondrial and 549 spliceosome function (Fig. 3b, Supplementary Data File 4); statistically enriched

550 biological cellular component terms in these functional categories are ribosomal proteins, components of the mitochondrial respiratory chain, two subunits of the histone $\mathrm{H} 4$ and snRNP Sm protein components of the spliceosome assembly. It is established that, in addition to influencing transcription, vitamin D can also influence post-transcriptional events by recruiting co-regulators. In this context it is relevant that components of the spliceosome such as snRNP Sm proteins that mediate both transcriptional control and splicing decisions, leading to alternatively spliced transcripts ${ }^{41}$, were upregulated by vitamin D supplementation in this study. These statistically significant gene expression changes were detected as temporal differences within each treatment group (differences at baseline (V1) versus 12 weeks after the intervention (V3) within single individuals). Differences in gene expression across treatment groups were only found in the South Asian group (Fig. $2 a, b)$. The SA treatment group that received supplementation with vitamin $D_{3}$, revealed three genes significantly upregulated and two down-regulated after the 12week intervention relative to the placebo group (Fig. 2; Supplementary Fig. 1). We note that the gene encoding cAMP-responsive element binding protein 1 (CREB1) is one of the three upregulated genes. It encodes a transcription factor that binds as a homodimer to the cAMP-responsive element. CREB1 protein is phosphorylated by several protein kinases, and induces transcription of genes in response to hormonal stimulation of the cAMP pathway. The protein kinase A (PKA) pathways are activated by the parathyroid hormone PTH in response to low serum calcium levels to maintain serum calcium homeostasis, primarily by promoting vitamin $D 1 \alpha-$

572 hydroxylation in the kidney. Both PTH and 1,25 $(\mathrm{OH})_{2}$ vitamin D have similar effects in promoting the maturation of osteocytes and opposing the differentiation of 
osteoblasts into osteocytes ${ }^{42,43}$. It is relevant to note in this context that all treatment groups in the SA cohort showed elevated serum PTH at the baseline sampling point relative to the WE cohort.

\section{Vitamins $D_{2}$ and $D_{3}$ do not influence expression of the same genes}

Our results indicate that the cellular responses to the two forms of vitamin D supplementation have some commonalities but also show some clear differences (catalogued in Supplementary Data File 4). For example, some biological processes such as histone modification and covalent chromatin modification are downregulated following vitamin $\mathrm{D}_{3}$ supplementation only, while spliceosomal function are upregulated by vitamin $D_{2}$ only. In light of the finding that vitamins $D_{2}$ and $D_{3}$ influence expression of different genes in the human blood transcriptome, recently we undertook a parallel in vitro study with a model rat cell line ${ }^{44}$. We examined the respective influences of the two physiologically active forms of vitamin $\mathrm{D}, 1,25(\mathrm{OH}) \mathrm{D}_{3}$ and $1,25(\mathrm{OH}) \mathrm{D}_{2}$ on differentiation and global gene expression in differentiating rat CG4 oligodendrocyte precursor cells and revealed considerable differences in the influence of the two types of vitamin $D$ on gene expression at $24 \mathrm{~h}$ and after $72 \mathrm{~h}$ following onset of differentiation. We demonstrated that $1,25(\mathrm{OH}) \mathrm{D}_{3}$ and $1,25(\mathrm{OH}) \mathrm{D}_{2}$ respectively influenced expression of 1,272 and 574 genes at $24 \mathrm{~h}$ following addition of the vitamin to the culture, where many of the changes in expression were specific to one or the other form of the vitamin ${ }^{44}$. This study provides evidence of the different direct effects of the two active vitamin $D$ metabolites on gene expression in vitro in cultured cells and provides some evidence that the changes we observed in the in vivo study may reflect the influence of the physiologically active forms of the vitamin, $1,25(\mathrm{OH}) \mathrm{D}_{3}$ and $1,25(\mathrm{OH}) \mathrm{D}_{2}$.

\section{Influence of vitamin D on expression of genes encoding immune pathways}

In common to both the $D_{2}$ and $D_{3}$ treatment groups, but not the placebo group, we found that many different pathways of the immune system are differentially expressed (largely down-regulated) by vitamin D, consistent with the emerging theme that one physiological role of vitamin $D$ is to suppress, or balance, the activity

607 of the immune system ${ }^{1,2}$ (Fig 3a, Fig. 4; Supplementary data files 3, 4 and 5). The 
608

609

610

611

612

613

614

615

616

617

618

619

620

621

622

623

624

625

626

627

628

629

630

631

632

633

634

635

636

637

638

639

640

641

immunomodulatory effects of vitamin D on both innate and adaptive immunity are well documented ${ }^{45-47}$. Vitamin $\mathrm{D}$ supplementation has the potential to ameliorate the symptoms of autoimmune diseases such as multiple sclerosis, as supported by several lines of evidence: the role in promoting differentiation of oligodendrocyte progenitor cells and therefore remyelination of CNS neurons ${ }^{48}$; suppression of the differentiation of potentially pathogenic T-cells and enhancement of regulatory T-cell differentiation ${ }^{49}$. However, a recent Cochrane review found little benefit of vitamin $D$ in this context ${ }^{50}$.

In this regard, the observation that vitamin $D_{2}$ and $D_{3}$ supplementation in the WE cohort were associated with divergent patterns of expression for interferon alpha (type 1) and interferon gamma (type 2) signature gene sets stands out as particularly interesting. Vitamin D appears to modulate type 1 interferon activity. For example, it enhances the effects of type 1 interferon treatment on mononuclear cells from patients with multiple sclerosis ${ }^{51}$, which parallels evidence for modest benefits of vitamin $D$ as an adjunct treatment with type 1 interferon in multiple sclerosis patients ${ }^{52,53}$. Vitamin D may also help suppress symptoms in autoimmune diseases such as systemic lupus erythematosus ${ }^{54}$, which are associated with chronic overactivity of interferon signalling and tentatively designated interferonopathies. Moreover, type 1 interferons play a critical role in defence against viral infections. Basal expression of type 1 interferon-stimulated genes, in the absence of infection, is key to priming a rapid and effective response to viral infection when it occurs ${ }^{55}$. There is currently intense interest in vitamin $D$ as both a potential prophylactic and a therapeutic agent for treatment of SARS-CoV-2 infection. One of the proposed modes of action of vitamin $D$ is modulation of interferon activity ${ }^{56}$; in this context our observation that vitamin $D_{3}$ (but not vitamin $D_{2}$ ) enhances the expression of genes involved in the interferon alpha response, is highly relevant to susceptibility to viral infection. Indeed, a recent genome-wide association study (GWAS) in 2,244 critically ill Covid-19 patients identified genetic variants leading to reduced interferon type 1 signalling that are associated with severe Covid-19 disease ${ }^{57}$.

We have also found differences in gene expression response to vitamin $D$ according to ethnicity (with the caveat that the sample size of the SA group was smaller than the WE group). Ethnic differences in response are suggested from the Weighted 
642 Gene Correlation Network Analysis (WGCNA) and the Gene Set Enrichment

643 Analysis (GSEA), where the response to vitamin $D_{3}$ intake appeared to have the

644 opposite effect on the type 1 and 2 interferon pathways in the SA group compared

645 with the WE group (Fig. 7). However, direct correlation between the stimulation of

646 immune responses, other than the interferon pathways, with an increase in serum

$64725(\mathrm{OH}) \mathrm{D}_{3}$ concentration was evident in the SA group only. This is the opposite of

648 that observed in the WE group where the effect of vitamin D supplementation was to

649 suppress several immune pathways.

650

\section{Conclusions}

652

653 The transcriptome results from this study differ from those of previous in vivo studies

654 which used supra-physiological doses of vitamin D over a short time-frame, or used

655 in vitro cultured cells. Indeed, where there is overlap in the identity of the

656 differentially expressed genes identified in the respective studies vitamin $D$ appears

657 to exert the opposite effect relative to our longer-term repeated physiological dose

658 study. Short-term responses to large boluses are relatively simple to do, and less

659 prone to noise, but their effect on gene expression does not seem to be consistent

660 with that observed with longer term repeated physiological dose studies like the

661 present study and therefore may not represent the real-world effects of repeated

662 dietary intake of vitamin D. Thus, despite the challenges and limitations of doing

663 longer term in vivo human studies, that are complicated by seasonal variation in

664 gene expression, inter-individual human variation, subtle physiological changes in

665 gene expression, more 'noise' through intake of other vitamin D sources during the

666 treatment intervention, our work suggests it may be important to conduct more such

667 longer term studies since the results from the shorter term interventions may not be

668 representative of the influence of vitamin D on normal physiological gene expression.

669 One potential criticism of whole blood transcriptome studies is that the results may

670 not represent the gene expression changes that occur in other tissues as a result of

671 vitamin D supplementation. However, we have shown, in comparable whole blood

672 transcriptome studies of human sleep physiology, that the changes in the human

673 blood transcriptome correlate with the changes that are observed in other tissues

674 and organs in comparable mammalian animal studies, giving us some confidence in 
675 the physiological relevance of the changes identified in this vitamin D transcriptome

676 study ${ }^{58,59}$.

678 The transcriptome results differ considerably between the two different ethnic

679 groups. While some of the differences may be attributable to differences in sample

680 sizes studied between the two groups it is also possible that some of the differences

681 represent genuine differences in the respective physiological responses of the two

682 ethnic groups. Alternatively, the differences may reflect the starting physiological

683 status of the SA participant group, who were found to have considerably lower

684 baseline vitamin D concentrations (and higher PTH concentrations) than the WE

685 group. The importance of clarifying these different responses is given particular

686 impetus by the emerging evidence for the interplay between ethnicity, skin tone,

687 vitamin D status and susceptibility to viral infection, Covid-19 being of particular

688 current relevance here ${ }^{3,4,60,61}$. The transcriptomic data presented in this study might

689 provide a useful context for further studies aimed at understanding the role of vitamin

$690 \mathrm{D}$ in influencing the immune response to SARS-CoV-2 infection, particularly in

691 relation to severe Covid-19. It is notable that the recent GenOMICC study of severe

692 Covid-19 disease highlighted a link between severe Covid-19 and reduced type 1

693 interferon signalling ${ }^{57}$. Our finding that vitamin $D_{3}$ appears to stimulate type 1

694 interferon signalling could be relevant in the context of its use as a prophylactic

695 treatment.

696

697

698 In summary our results indicate that whilst the two forms of vitamin $D\left(D_{2}\right.$ and $\left.D_{3}\right)$

699 exert some overlapping roles in human physiology, each form may elicit different

700 molecular responses, findings which warrant a more detailed consideration of the

701 system-wide effects of these two forms vitamin D. Clearly, this type of study requires

702 replication, using a much larger independent cohort, with balanced representation

703 from different ethnic groups. This is perhaps of particular importance to at-risk ethnic

704 groups, including black and South Asian populations who reside in northern

705 latitudes. The studies would need to be time course-based (temporal) to track gene

706 expression changes within individuals from baseline activity to defined sampling

707 times and would need in-built control to account for seasonal gene expression

708 changes. They would need to be designed specifically to answer whether different 
ethnicity or different vitamin D baseline levels give rise to different responses to

710 vitamin D supplementation.

712 Since some pathways appear to be regulated specifically by vitamin $D_{3}$, or in some

713 cases, in opposing directions by vitamin $D_{3}$ and $D_{2}$, future studies should investigate

714 whether vitamin $D_{2}$ supplementation might counteract some of the benefits of vitamin

$715 D_{3}$ on human health. This possibility is prompted by the findings from this cohort that

716 the circulating concentration of $25(\mathrm{OH}) \mathrm{D}_{3}$ within vitamin $\mathrm{D}_{2}$-treated participants was

717 significantly lower after the 12-week intervention than in the placebo group who

718 received no vitamin D supplements - suggesting that the former might be depleted

719 by the latter. The results from this study suggest that guidelines on food fortification

720 and supplementation with specific forms of vitamin D may need revisiting.

\section{Methods}

\section{Blood Transcriptome analysis}

725 The recruitment of individuals as part of the BBSRC D2-D3 Study was described

726 previously ${ }^{24}$. This study received ethical approval from the South-East Coast

727 (Surrey) National Health Service Research Ethics Committee (11/LO/0708) and the

728 University of Surrey Ethics Committee (EC/2011/97/FHMS). All of the participants

729 gave written informed consent in agreement with the Helsinki Declaration before

730 commencing study activities. Briefly, 335 women of both South Asian (SA) and white

731 European (WE) descent were randomised to one of three intervention groups for 12-

732 weeks and provided with daily doses of vitamin $D$ within fortified foods: placebo, 15

$733 \mu \mathrm{g} / \mathrm{d}$ vitamin $D_{2}$ or $15 \mu \mathrm{g} / \mathrm{d}$ vitamin $D_{3}$. Of the 335 participants, 97 were selected for

734 transcriptome analysis (32 were WE, 32 were A and 33 were selected at random

735 from both ethnic groups to form a balanced placebo group). High responders (>50\%

736 increase in 25OHD concentrations) and low responders ( $<50 \%$ increase in $250 H D$

737 concentrations) were included within both vitamin $D_{2}$ and $D_{3}$ supplementation groups

738 to maximize the chance of observing differences in gene expression among

739 subjects. The selected time points for transcriptome analysis were $\mathrm{V} 1$ representing

740 the baseline and V3, 12 weeks after treatment commenced. 
742 Whole peripheral blood $(2.5 \mathrm{ml})$ was collected using PAXgene Blood RNA tubes

743 (Becton Dickinson). PAXgene Blood RNA Tubes were inverted ten times

744 immediately after drawing blood, stored upright at $15-25^{\circ} \mathrm{C}$ for 24 hours, followed by

745 a $-20^{\circ} \mathrm{C}$ freezer for 24 hours and then into a $-80^{\circ} \mathrm{C}$ freezer for long-term storage.

747 Transcriptomic analysis was conducted essentially as described in previous studies

74858,59 . Total RNA was isolated using the PAXgene Blood RNA Kit (Qiagen) following

749 the manufacturers recommendations. cRNA was synthesised and fluorescently

750 labelled with Cy3-CTP from 200 ng of total RNA using the Low Input QuickAmp

751 Labelling Kit, One Color (Agilent Technology). Labelled cRNA was hybridised on a

752 Sure Print G3 Human Gene Expression 8 x 60K v2 microarray slide (Agilent

753 Technologies). Standard manufacturer's instructions for one colour gene-expression

754 analysis were followed for labelling, hybridisation and washing steps. Extracted RNA

755 was quantified using NanoDrop ND2000 spectrophotometer (Thermo Scientific).

756 RNA quality and integrity was evaluated using either the Bioanalyzer 2100 or the

757 TapeStation 4200 (Agilent Technologies). Only RNA samples with an RNA Integrity

758 Number (RIN) of $>7.0$ were subjected to DNA microarray analysis. Microarrays were

759 hybridised at $65^{\circ} \mathrm{C}$ for 17 hours in an Agilent Hybridization Oven on a rotisserie at 10

$760 \mathrm{rpm}$. The washed microarrays were scanned using an Agilent Microarray Scanner

761 with a resolution of $2 \mu \mathrm{m}$.

Transcriptome data processing and differential expression analysis.

764 Raw scanned microarray images were processed using Agilent Feature Extraction

765 software (v11.5.1.1) with the Agilent 039494_D_F_20140326_human_8x60K_v2

766 grid, and then imported into $R$ for normalization and analysis using the LIMMA

767 package ${ }^{62}$. Microarray data were background-corrected using the 'normexp' method

768 (with an offset of 50) and quantile normalised, producing expression values in the log

769 base 2 scale. The processed data were then filtered to remove probes exhibiting low

770 signals across the arrays, retaining non-control probes that are at least $10 \%$ brighter

771 than negative control probe signals on at least 41 arrays ( $20 \%$ of the arrays in the

772 analysis). Data from identical replicate probes were then averaged to produce

773 expression values at the unique probe level. Initial data exploration identified one

774 sample (participant 0017, V3 time point) with array data that was a notable outlier

775 from the group and therefore both the V1 and V3 microarrays for this subject were 
excluded from all subsequent analysis, reprocessing the data as above in their absence before proceeding.

Tests for differential expression were performed using LIMMA, applying appropriate linear model designs to identify: (i) significant differences in the transcriptional responses occurring across the 12-week V1 to V3 period of the study between the treatment and placebo groups for each ethnic cohort (example contrasts tested, in the format ethnicity_treatment_time: (WE_D2_V3 - WE_D2_V1) - (WE_P_V3-

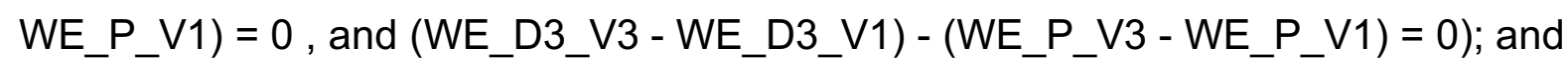
ii) to determine significant changes for each ethnic cohort within each treatment group between the $\mathrm{V} 3$ and $\mathrm{V} 1$ sampling points, blocking on subject identity (example contrasts tested: WE_D2_V3 - WE_D2_V1 = 0, WE_D3_V3 - WE_D3_V1 = 0). Blocking on subject identity was used to control for inter-subject variability. Significance $p$-values were corrected for multiplicity using the Benjamini and Hochberg method, obtaining adjusted p-values (adj.P.Val). Normalised data for all participants (V3 - V1) was assessed by principal component analysis to screen for any batch effects (Supplementary Fig. 3).

\section{Functional enrichment and network analysis}

Functional enrichment analysis of lists of genes of interest possessing valid ENTREZ gene identifiers was performed using the $R$ package clusterProfiler ${ }^{63}$. The software produces adjusted p-values (p.adjust) using the Benjamini and Hochberg correction method. Construction and analysis of protein-protein interaction networks from sets of genes was undertaken in Cytoscape ${ }^{64}$ using the STRING plugin ${ }^{65}$. Cytoscape was also used to construct and visualise commonality in the functional categories identified as being significantly enriched in the genes responding to the experimental treatments. To visualise categories identified from the $D_{2}$ or $D_{3}$ treatment groups but not from the placebo (e.g. Figs. 4 and 5) significantly enriched categories (p.adjust < $0.01)$ from all groups were imported such that treatment group nodes $\left(D_{2}, D_{3}\right.$ and placebo $(P))$ are linked to functional category nodes by edges assigned the corresponding p.adjust values. All nodes with an edge connection to the placebo treatment node were then removed and the resulting networks further filtered to retain only those nodes with at least one edge connection with $p$.adjust $<=0.001$. 
810 Weighted gene co-expression network analysis was performed in $\mathrm{R}$ using the

811 WGCNA ${ }^{37}$ and CoExpNets ${ }^{66}$ packages. A normalised expression data matrix

812 generated by filtering to retain probes with signals more markedly above background

813 (30\% brighter than negative control probe signals on at least 41 arrays) was used as

814 input, consisting of 12,169 unique probes. Signed scale-free networks were

815 constructed separately for the data for the SA and WE ethnic groups using 50

816 iterations of the k-means clustering option in the CoExpNets

817 'getDownstreamNetwork' function to refine the clustering process (Supplementary

818 Data File 6). Pearson correlations between cluster module eigengenes and

819 metadata for serum $25(\mathrm{OH}) \mathrm{D}_{2}, 25(\mathrm{OH}) \mathrm{D}_{3}$, total $25(\mathrm{OH}) \mathrm{D}$ and PTH concentrations

820 were calculated using pairwise complete observations.

\section{Gene set enrichment analysis (GSEA)}

823 GSEA was performed using the R package clusterProfiler ${ }^{63}$, applying the default

824 parameters which implement the fgsea algorithm and correct for multiple testing 825 using the Benjamini and Hochberg method. Gene sets were obtained from MSigDB

826 via the msigdbr package ${ }^{67}$. Ranked gene lists for interrogation were derived from

827 LIMMA analysis of the unfiltered microarray data, pre-ranking genes according to 828 their t-statistic.

830 Data availability: All microarray data are available in the ArrayExpress database

831 (http://www.ebi.ac.uk/arrayexpress) under accession number E-MTAB-8600.

\section{Acknowledgements}

834 This work was funded by BBSRC (UK) DRINC award BB/I006192/1 to SLN and 835 CPS, by a University of Brighton Innovation Seed Fund Award (to CPS). The authors 836 would like to particularly thank Michael Chowen CBE DL and Maureen Chowen for 837 their generous philanthropic donation (to CPS).

\section{Declaration of interests}

840 All authors confirm that they have no conflicts of interest. 
Figure 1. (a) Selection of 98 subject participants from the Tripkovic et al. (2017) study ${ }^{24}$ for transcriptomic analysis of their V1 (baseline) and V3 (12-week) samples in the present study; (b) Ethnicity and treatment group membership for the 97 subjects for which transcriptomic data was obtained (data for one South Asian subject from the placebo group did not pass quality control); (c) Metadata on serum concentrations of $25(\mathrm{OH}) \mathrm{D}_{2}, 25(\mathrm{OH}) \mathrm{D}_{3}$, total $25(\mathrm{OH}) \mathrm{D}, \mathrm{PTH}$ and calcium (albuminadjusted) for the 97 subjects (see Supplementary Data File 1 for details). Participants were selected to provide comparable numbers between the placebo and the two vitamin $D$ treatment groups, covering the full range of serum responses to supplementation within the $D_{2}$ and $D_{3}$ treatment groups, as judged from the measured changes in serum $25(\mathrm{OH}) \mathrm{D}_{2}$ or $25(\mathrm{OH}) \mathrm{D}_{3}$ concentrations between $\mathrm{V} 1$ and V3.

Figure 2. (a) Summary of limma differential expression test results identifying significant changes (adj.P.Val<=0.05) in transcript probe abundance within and between the experimental groups (see Supplementary Data File 2 for full details). Both the number of unique microarray probes, and the number of unique genome loci they represent, are indicated. WE = "white European"; SA = "South Asian"; D2 = vitamin $D_{2}$ treatment group; $D 3=$ vitamin $D_{3}$ treatment group; $P=$ placebo group. (b) The CREB1 gene probe signal is significantly different (adj.P.Val=0.021) between the vitamin $D_{3}$-treated group and the placebo group over the course of the $\mathrm{V} 1$ to $\mathrm{V} 3$ study period in the South Asian cohort (i.e. [SA D3 V3 v V1] v [SA P V3 v V1] comparison from Fig. 2a). (c) Venn diagrams showing the numbers of probes in the white European (WE) cohort that are specifically significantly (adj.P.Val<=0.05) down- or up-regulated in V3 compared to V1 in each treatment group. (d) Comparison of the $\log _{2}$ fold-changes (FC) in abundance occurring from V1 to V3 in the WE cohort in the $D_{2}$ and $D_{3}$ treatment groups. Probes significantly up-regulated in both $D_{2}$ and $D_{3}$ groups but not the placebo are coloured red, while those similarly down-regulated are shown in blue (see Supplementary Data File 3). A probe detecting SEC14L1 shows the largest decreases in abundance in those downregulated by $D_{2}$ and $D_{3}$ but not placebo. 
878 Figure 3. Protein-protein interaction networks for gene products corresponding to 879 the probes (a) significantly down-regulated or (b) significantly up-regulated in both 880 the $D_{2}$ and $D_{3}$ treatment groups of the WE cohort, but not the placebo group. Details 881 given in Supplementary Data File 3. The networks were generated using the 882 STRING database of Homo sapiens medium confidence (0.4) interactions, and only 883 connected nodes are shown. Networks for both (a) and (b) are significantly enriched 884 for interactions compared to randomised sets, yielding $p$-values of $1.98 \times 10^{-6}$ and $8854.99 \times 10^{-9}$, respectively.

887 Figure 4. Networks illustrating the functional categories significantly enriched in the gene products represented by the probes significantly down-regulated (adj.P.Val $\leq$

$8890.05)$ in the $D_{2}$ and $D_{3}$ treatment groups of the WE cohort, but not in the placebo 890 group. Gene products represented by the significantly down-regulated probes in the

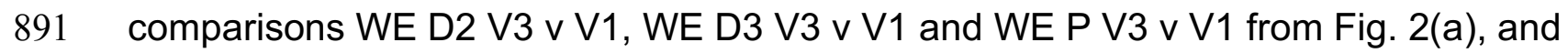
892 possessing ENTREZ identifiers, were subjected separately to functional enrichment 893 analysis using compareCluster ${ }^{63}$. The details for each group are given in

894 Supplementary Data File 4. Significantly enriched categories ( $p$.adjust $\leq 0.01$ ) from 895 all groups were imported into Cytoscape ${ }^{64}$ to visualise categories identified from the $896 D_{2}$ or $D_{3}$ treatment groups but not by the placebo (see Methods for details). The

897 thickness of lines (edges) drawn to connect the nodes shown increases with

898 increasing significance (p.adjust-values) and only categories where at least one 899 edge is $\leq 1 \times 10^{-3}$ are shown. The complete networks for each differentially 900 expressed group of genes are shown in Supplementary Data File 5.

902 Figure 5. Networks illustrating the functional categories significantly enriched in the 903 gene products represented by the probes significantly up-regulated (adj.P.Val $\leq$ 9040.05 ) in the $D_{2}$ and $D_{3}$ treatment groups of the WE cohort, but not in the placebo 905 group. Gene products represented by the significantly up-regulated probes in the 906 comparisons WE D2 V3 $\vee$ V1, WE D3 V3 $\vee$ V1 and WE P V3 $\vee$ V1 from Fig. 2(a), and 907 possessing ENTREZ identifiers, were subjected separately to functional enrichment 908 analysis using compareCluster as detailed in the legend to Fig. 4. The complete 909 networks for each differentially expressed group of genes are shown in

\section{Supplementary Data File 5.}


912 Figure 6. Gene co-expression networks for the WE (a) and SA (b) ethnic groups. For

913 each group of subjects, signed scale-free networks were constructed from the

914 expression data using WGCNA to cluster probes with similar expression

915 characteristics across all samples into discrete modules (colours on the y-axis:

916 colours in (a) and (b) are independent). Module membership for the modules of

917 genes identified in the WGCNA analysis of the data from the South Asian and white

918 European cohorts are detailed in Supplementary Data File 6. The colour scale to

919 the right of each panel represents the Pearson correlation (from -1 to +1 ) between

920 the expression profile for each module's eigengene and the respective serum

$92125(\mathrm{OH}) \mathrm{D}_{2}, 25(\mathrm{OH}) \mathrm{D}_{3}$, total 25(OH)D and PTH concentrations (x-axis). The Pearson

922 correlation coefficients are also provided in each box, followed in brackets by an

923 adjusted $p$-value, testing for the significance of each correlation. Statistically

924 significant correlations (adjusted $p$-value $\leq 0.05$ ) are circled in red. A headline

925 significant GO functional enrichment category (p.adjust $\leq 0.01$ ) for each significant

926 module is shown to the right; GO test results for each module are summarised in

927 Supplementary Data Files 7 and 8).

929 Figure 7. Gene Set Enrichment Analysis using the MSigDB hallmark gene sets

930 indicates divergent behaviour for the interferon alpha and gamma response gene

931 sets following supplementation with vitamin $D_{2}$ and $D_{3}$ in the WE cohort. Coloured

932 tiles in the heatmap correspond to gene sets exhibiting a statistically significant

933 (padjust<=0.05), concordant change between the V3 and V1 sampling times for the

934 placebo $(P)$, vitamin $D_{2}(D 2)$ or vitamin $D_{3}(D 3)$ treatment groups in the SA or WE

935 cohorts. Grey tiles indicate non-significance. A positive normalised enrichment score

936 (NES) indicates up-regulation of a gene set in V3 relative to $\mathrm{V} 1$, and conversely

937 down-regulation is indicated by a negative NES score. Full results are provided in

938 Supplementary Data File 9, and the behavior of the leading edge, core genes

939 accounting for the significant enrichment signals in the interferon alpha and gamma

940 response sets are illustrated in Supplementary Figs. 4-6. 


\section{Supplementary Figure 1.}

945 Summary of the probes identified as being significantly different in the 'difference-in-

946 difference' analysis of the SA cohort (comparison [SA D3 V3 v V1] v [SA P V3 v V1]

947 in Fig. 2a). (A) Differential expression test results; (B) Probe signal abundance $\left(\log _{2}\right)$

948 distribution across the experimental treatments in the SA cohort.

\section{Supplementary Figure 2.}

951 Seasonal gene expression as a feature of the 12-week study $(P=$ placebo, $D 2=$

952 vitamin $D_{2}, D 3=$ vitamin $D_{3}$ ). (a) Summary of the timing of the $V 1$ samples taken in

953 the study (for each individual, V3 samples were taken 12 weeks after the V1

954 sample); (b) Significant over-representation of the seasonal genes identified by

955 Dopico et al (2015) Nat Commun, 6, 7000 (BABYDIET dataset) in the genes

956 identified as significantly changing in the white European (WE) placebo, $D_{2}$ and $D_{3}$

957 treatment groups.

958

\section{Supplementary Figure 3.}

960 Principal component analysis of the transcriptome data for the 97 subjects passing

961 quality control. Data for samples from visits V1 and V3 are processed as the

962 difference between V3 and V1 (V3 minus V1) for each subject. The distribution is

963 relatively random.

964

\section{Supplementary Figure 4.}

966 The MSigDB Hallmark gene sets "Interferon alpha response" and "Interferon gamma

967 response" show statistically significant, concordant up-regulation between the V3

968 and V1 sampling times following supplementation with vitamin $\mathrm{D}_{3}$ in the WE cohort

969 (adjusted p-values 9.36e-15 and 1.59e-11, respectively). a) Leading edge, core

970 genes accounting for the enrichment signals in the gene set enrichment analysis of

971 the $D_{3}$ data. b) Heatmap showing the log2 fold change between V3 and V1 for each

972 gene identified in a), for all the treatment groups in both the SA and WE cohorts $(P=$ 973 placebo, $\mathrm{D} 2=$ vitamin $\mathrm{D}_{2}, \mathrm{D} 3=$ vitamin $\left.\mathrm{D}_{3}\right)$. Gene membership of the Hallmark

974 interferon alpha and gamma response gene sets is indicated to the right. Genes 
shared between both sets are labelled "alpha \& gamma". Gene PARP14 and ISG20

976 belong to both sets, but are leading edge genes only for the alpha response.

977

978 Supplementary Figure 5.

979 The MSigDB Hallmark gene sets "Interferon alpha response" and "Interferon gamma 980 response" show statistically significant, concordant down-regulation between the V3 981 and V1 sampling times following supplementation with vitamin $D_{2}$ in the WE cohort 982 (adjusted p-values 4.22e-02 and 1.61e-04 respectively). a) Leading edge, core 983 genes accounting for the enrichment signals in the gene set enrichment analysis. b) 984 Heatmap showing the log2 fold change between V3 and V1 for each gene identified 985 in a), for all the treatment groups in both the SA and WE cohorts ( $P=$ placebo, D2 = 986 vitamin $D_{2}$, D3 = vitamin $D_{3}$ ). Gene membership of the Hallmark interferon alpha and 987 gamma response gene sets is indicated to the right. Genes shared between both 988 sets are labelled "alpha \& gamma". Gene PARP14 belongs to both sets, but is a 989 leading edge gene only for the alpha response.

990

\section{Supplementary Figure 6.}

992 The MSigDB Hallmark gene sets "Interferon alpha response" and "Interferon gamma 993 response" show statistically significant, concordant down-regulation between the V3 994 and V1 sampling times following supplementation with vitamin $D_{3}$ in the SA cohort 995 (adjusted p-values 1.34e-09 and 8.45e-09 respectively). (a) Leading edge, core 996 genes accounting for the enrichment signals in the gene set enrichment analysis. (b) 997 Heatmap showing the $\log _{2}$ fold change between V3 and V1 for each gene identified 998 in (a), for all the treatment groups in both the SA and WE cohorts $(P=$ placebo, D2 = 999 vitamin $D_{2}, D 3=$ vitamin $D_{3}$ ). Gene membership of the Hallmark interferon alpha and 1000 gamma response gene sets is indicated to the right. Genes shared between both 1001 sets are labelled "alpha \& gamma". Gene PSMB8 belongs to both sets, but is a 1002 leading edge gene only for the alpha response.

1003 


\section{Supplementary Data File 1}

1007 Metadata on serum concentrations of $25(\mathrm{OH}) \mathrm{D}_{2}, 25(\mathrm{OH}) \mathrm{D}_{3}$, total $25(\mathrm{OH}) \mathrm{D}, \mathrm{PTH}$ and

1008 calcium (albumin-adjusted) for the 97 subjects subjected to transcriptomic analysis

1009 (selected from the cohort described by Tripkovic et al. 2017).

1010

1011 Supplementary Data File 2 [Note: data file is 9.1 MB.]

1012 Significant differences in the transcriptional responses occurring across the 12-week

1013 V1 to V3 period of the study between the vitamin $D_{2}$, vitamin $D_{3}$ and placebo

1014 treatment groups for each ethnic cohort, and significant changes within each group

1015 between the V3 and V1 sampling points (see Fig. 2).

1016

1017 Supplementary Data File 3

1018 Functional analysis of the genes represented by the probes specifically repressed by

$1019 D_{2}$ and $D_{3}$ in the white European cohort (blue data points in Fig. 2d) or induced by $D_{2}$

1020 and $D_{3}$ in the white European cohort (red data points in Fig. 2d).

1021

1022 Supplementary Data File 4

1023 Comparative functional enrichment analysis of all significant changes in each

1024 treatment group in the white European cohort (Fig. 2c; and see Figs 4 and 5).

1025

\section{Supplementary Data File 5}

1027 Networks illustrating all the functional categories significantly enriched (p.adjust <

1028 0.01) in analysis of the gene products represented by the probes significantly up- or

1029 down-regulated (adj.P.Val < 0.05) in the white European cohort treatment groups

1030 (from data presented in Supplementary Data File 4).

1031

1032 Supplementary Data File 6

1033 Module membership for the modules of genes identified in the WGCNA analysis of

1034 the data from the South Asian and white European cohorts, as presented in Fig. 6.

\section{Supplementary Data File 7}


1037 Gene ontology functional enrichment analysis results for the modules of genes

1038 identified in the WGCNA analysis of the data from the white European cohort.

1039

1040 Supplementary Data File 8

1041 Gene ontology functional enrichment analysis results for the modules of genes

1042 identified in the WGCNA analysis of the data from the South Asian cohort.

1043

1044 Supplementary Data File 9

1045 Gene Set Enrichment Analysis results for the analysis reported in Fig. 7.

1046

1047 


\section{References}

10511 Prietl, B., Treiber, G., Pieber, T. R. \& Amrein, K. Vitamin D and immune function. Nutrients 5, 1052 2502-2521, doi:10.3390/nu5072502 (2013).

10532 Martens, P. J., Gysemans, C., Verstuyf, A. \& Mathieu, A. C. Vitamin D's Effect on Immune $1054 \quad$ Function. Nutrients 12, doi:10.3390/nu12051248 (2020).

10553 Kohlmeier, M. Avoidance of vitamin D deficiency to slow the COVID-19 pandemic. BMJ

1056 Nutrition, Prevention \& Health 3, doi:10.1136/bmjnph-2020-000096 (2020).

10574 Lanham-New, S. A., Webb, A. R., Cashman, K. D. \& al, e. Vitamin D and SARS-CoV-2

1058 virus/COVID-19 disease. BMJ Nutrition, Prevention \& Health 3, doi:10.1136/bmjnph-2020-

$1059000089(2020)$.

10605 Holick, M. F. Vitamin D deficiency. N Engl J Med 357, 266-281, doi:10.1056/NEJMra070553

1061 (2007).

10626 Bouillon, R. Extra-Skeletal Effects of Vitamin D. Front Horm Res 50, 72-88,

1063 doi:10.1159/000486072 (2018).

10647 Zhang, Y. et al. Association between vitamin D supplementation and mortality: systematic 1065 review and meta-analysis. British Medical Journal 366, 14673, doi:10.1136/bmj.14673 (2019).

10668 Long, M. D., Sucheston-Campbell, L. E. \& Campbell, M. J. Vitamin D receptor and RXR in the 1067 post-genomic era. J Cell Physiol 230, 758-766, doi:10.1002/jcp.24847 (2015).

10689 Hollis, B. W. Comparison of equilibrium and disequilibrium assay conditions for 1069 ergocalciferol, cholecalciferol and their major metabolites. Journal of steroid biochemistry $1070 \quad$ 21, 81-86, doi:10.1016/0022-4731(84)90063-3 (1984).

107110 Houghton, L. A. \& Vieth, R. The case against ergocalciferol (vitamin D2) as a vitamin 1072 supplement. The American journal of clinical nutrition 84, 694-697, 1073 doi:10.1093/ajcn/84.4.694 (2006).

107411 Tripkovic, L. et al. Comparison of vitamin D2 and vitamin D3 supplementation in raising 1075 serum 25-hydroxyvitamin D status: a systematic review and meta-analysis. The American journal of clinical nutrition 95, 1357-1364, doi:10.3945/ajcn.111.031070 (2012). health issue: using vitamin D2 or vitamin D3 in future fortification strategies. Proc Nutr Soc

108013 Biancuzzo, R. M., Holick, M. F. \& Winter, M. R. Reply to S Lanham-New et al. The American $1081 \quad$ journal of clinical nutrition 92, 999-1000 (2010). 

effective as an oral supplement in maintaining vitamin $\mathrm{D}$ status in adults. The American journal of clinical nutrition 91, 1621-1626, doi:10.3945/ajcn.2009.27972 (2010).

1085 Lanham-New, S., Vieth, R. \& Heaney, R. Vitamin D2 and vitamin D3 comparisons:

1086

1087 fundamentally flawed study methodology. The American journal of clinical nutrition 92, 999;

1088 author reply 999-1000, doi:10.3945/ajcn.2010.30099 (2010).

1089 ODS, N. Vitamin $D,<$ https://ods.od.nih.gov/factsheets/VitaminD-HealthProfessional/>

1090 (2020).

1091

Pilz, S. et al. Vitamin D testing and treatment: a narrative review of current evidence. Endocr Connect 8, R27-R43, doi:10.1530/EC-18-0432 (2019).

$$
\text { Schwalfenberg, G. K. A review of the critical role of vitamin } D \text { in the functioning of the }
$$
immune system and the clinical implications of vitamin D deficiency. Mol Nutr Food Res 55, 96-108, doi:10.1002/mnfr.201000174 (2011). doi:10.1152/physrev.00014.2015 (2016). Christakos, S., Dhawan, P., Verstuyf, A., Verlinden, L. \& Carmeliet, G. Vitamin D: Metabolism, Molecular Mechanism of Action, and Pleiotropic Effects. Physiol Rev 96, 365-408, Rosen, C. J. et al. The nonskeletal effects of vitamin D: an Endocrine Society scientific statement. Endocr Rev 33, 456-492, doi:10.1210/er.2012-1000 (2012).

110523 Zittermann, A., Ernst, J. B., Gummert, J. F. \& Borgermann, J. Vitamin D supplementation, 1106 body weight and human serum 25-hydroxyvitamin D response: a systematic review.

1107 European journal of nutrition 53, 367-374, doi:10.1007/s00394-013-0634-3 (2014).

110824 Tripkovic, L. et al. Daily supplementation with 15 mug vitamin D2 compared with vitamin D3 1109 to increase wintertime 25-hydroxyvitamin D status in healthy South Asian and white 1110 European women: a 12-wk randomized, placebo-controlled food-fortification trial. The 1111 American journal of clinical nutrition 106, 481-490, doi:10.3945/ajcn.116.138693 (2017).

111225 Holick, M. F. et al. Vitamin D2 is as effective as vitamin D3 in maintaining circulating concentrations of 25-hydroxyvitamin D. The Journal of clinical endocrinology and metabolism 93, 677-681, doi:10.1210/jc.2007-2308 (2008). 

supplementation in infants: a systematic review and meta-analysis of clinical intervention trials. European journal of nutrition 59, 359-369, doi:10.1007/s00394-019-01912-x (2020). Berlanga-Taylor, A. J. et al. Genomic Response to Vitamin D Supplementation in the Setting of a Randomized, Placebo-Controlled Trial. EBioMedicine 31, 133-142, doi:10.1016/j.ebiom.2018.04.010 (2018).

$112128 \quad$ Neme, A. et al. In vivo transcriptome changes of human white blood cells in response to vitamin D. The Journal of steroid biochemistry and molecular biology 188, 71-76,

112429 Hossein-nezhad, A., Spira, A. \& Holick, M. F. Influence of vitamin D status and vitamin D3 supplementation on genome wide expression of white blood cells: a randomized doubleblind clinical trial. PloS one 8, e58725, doi:10.1371/journal.pone.0058725 (2013). Zhang, R. et al. Transcriptional regulation of BMP2 expression by the PTH-CREB signaling pathway in osteoblasts. PloS one 6, e20780, doi:10.1371/journal.pone.0020780 (2011). Dopico, X. C. et al. Widespread seasonal gene expression reveals annual differences in human immunity and physiology. Nat Commun 6, 7000, doi:10.1038/ncomms8000 (2015). Sonbul, S. N. et al. Saccharomyces cerevisiae-like 1 (SEC14L1) is a prognostic factor in breast cancer associated with lymphovascular invasion. Mod Pathol 31, 1675-1682, doi:10.1038/s41379-018-0092-9 (2018). Agell, L. et al. A 12-gene expression signature is associated with aggressive histological in prostate cancer: SEC14L1 and TCEB1 genes are potential markers of progression. Am J Pathol 181, 1585-1594, doi:10.1016/j.ajpath.2012.08.005 (2012). Burdelski, C. et al. Saccharomyces cerevisiae-like 1 overexpression is frequent in prostate cancer and has markedly different effects in Ets-related gene fusion-positive and fusionnegative cancers. Hum Pathol 46, 514-523, doi:10.1016/j.humpath.2014.06.006 (2015). Szklarczyk, D. et al. The STRING database in 2017: quality-controlled protein-protein association networks, made broadly accessible. Nucleic Acids Research 45, D362-D368, doi:10.1093/nar/gkw937 (2017).

Fetahu, I. S., Hobaus, J. \& Kallay, E. Vitamin D and the epigenome. Front Physio/ 5, 164, doi:10.3389/fphys.2014.00164 (2014).

Langfelder, P. \& Horvath, S. WGCNA: an R package for weighted correlation network analysis. BMC Bioinformatics 9, 559, doi:10.1186/1471-2105-9-559 (2008). Koivisto, O., Hanel, A. \& Carlberg, C. Key Vitamin D Target Genes with Functions in the Immune System. Nutrients 12, doi:10.3390/nu12041140 (2020). 
114939 Bouchon, A., Facchetti, F., Weigand, M. A. \& Colonna, M. TREM-1 amplifies inflammation

1150 and is a crucial mediator of septic shock. Nature 410, 1103-1107, doi:10.1038/35074114

$1151 \quad$ (2001).

115240 Hatzi, K. et al. A hybrid mechanism of action for BCL6 in B cells defined by formation of

1153 functionally distinct complexes at enhancers and promoters. Cell Rep 4, 578-588,

1154 doi:10.1016/j.celrep.2013.06.016 (2013).

115541 Zhou, R. et al. Vitamin D and alternative splicing of RNA. The Journal of steroid biochemistry

1156 and molecular biology 148, 310-317, doi:10.1016/j.jsbmb.2014.09.025 (2015).

115742 St John, H. C. et al. The parathyroid hormone-regulated transcriptome in osteocytes: parallel

1158 actions with 1,25-dihydroxyvitamin D3 to oppose gene expression changes during

1159 differentiation and to promote mature cell function. Bone 72, 81-91,

1160 doi:10.1016/j.bone.2014.11.010 (2015).

116143 St John, H. C. et al. The osteoblast to osteocyte transition: epigenetic changes and response 1162 to the vitamin D3 hormone. Mol Endocrinol 28, 1150-1165, doi:10.1210/me.2014-1091

1163 (2014)

116444 Mengozzi, M., Hesketh, A., Bucca, G., Ghezzi, P. \& Smith, C. P. Vitamins D3 and D2 have 1165 marked but different global effects on gene expression in a rat oligodendrocyte precursor

1166

1167 cell line. Molecular medicine (Cambridge, Mass.) 26, 32, doi:10.1186/s10020-020-00153-7

116845 Liu, P. T. et al. Toll-like receptor triggering of a vitamin D-mediated human antimicrobial 1169 response. Science 311, 1770-1773, doi:10.1126/science.1123933 (2006).

117046 Hayes, C. E. \& Nashold, F. E. Vol. 2 (ed D. Feldman) 989-1024 (Elsevier Inc., 2018).

117147 Teles, R. M. et al. Type I interferon suppresses type II interferon-triggered human anti-

1172 mycobacterial responses. Science 339, 1448-1453, doi:10.1126/science.1233665 (2013).

$117348 \quad$ Gomez-Pinedo, U. et al. Vitamin D increases remyelination by promoting oligodendrocyte

1174 lineage differentiation. Brain Behav 10, e01498, doi:10.1002/brb3.1498 (2020).

117549 Wasnik, S., Sharma, I., Baylink, D. J. \& Tang, X. Vitamin D as a Potential Therapy for Multiple

1176 Sclerosis: Where Are We? International journal of molecular sciences 21,

1177 doi:10.3390/ijms21093102 (2020).

117850 Jagannath, V. A. et al. Vitamin D for the management of multiple sclerosis. Cochrane 1179 Database Syst Rev 9, CD008422, doi:10.1002/14651858.CD008422.pub3 (2018).

$118051 \quad$ Feng, X. et al. Vitamin D enhances responses to interferon-beta in MS. Neurol Neuroimmunol 1181 Neuroinflamm 6, e622, doi:10.1212/NXI.0000000000000622 (2019). 
$118252 \quad$ Hupperts, R. et al. Randomized trial of daily high-dose vitamin D3 in patients with RRMS

1183 receiving subcutaneous interferon beta-1a. Neurology 93, e1906-e1916,

1184 doi:10.1212/WNL.0000000000008445 (2019).

$118553 \quad$ Camu, W. et al. Cholecalciferol in relapsing-remitting MS: A randomized clinical trial

1186 (CHOLINE). Neurol Neuroimmunol Neuroinflamm 6, doi:10.1212/NXI.0000000000000597

1187 (2019).

$118854 \quad$ Zheng, R. et al. Efficacy and Safety of Vitamin D Supplementation in Patients With Systemic

1189 Lupus Erythematosus: A Meta-analysis of Randomized Controlled Trials. Am J Med Sci 358,

1190 104-114, doi:10.1016/j.amjms.2019.04.020 (2019).

$119155 \quad$ Mostafavi, S. et al. Parsing the Interferon Transcriptional Network and Its Disease

$1192 \quad$ Associations. Cell 164, 564-578, doi:10.1016/j.cell.2015.12.032 (2016).

119356 Gauzzi, M. C. \& Fantuzzi, L. Reply to Jakovac: COVID-19, vitamin D, and type I interferon. Am 1194 J Physiol Endocrinol Metab 319, E245-E246, doi:10.1152/ajpendo.00315.2020 (2020).

119557 Pairo-Castineira, E. et al. Genetic mechanisms of critical illness in Covid-19. Nature, doi:10.1038/s41586-020-03065-y (2020).

Archer, S. N. et al. Mistimed sleep disrupts circadian regulation of the human transcriptome.

1198 Proceedings of the National Academy of Sciences of the United States of America 111, E682691, doi:10.1073/pnas.1316335111 (2014).

1200

59

Moller-Levet, C. S. et al. Effects of insufficient sleep on circadian rhythmicity and expression

1201 amplitude of the human blood transcriptome. Proceedings of the National Academy of

1202 Sciences of the United States of America 110, E1132-1141, doi:10.1073/pnas.1217154110

1203 (2013)

$120460 \quad$ Kmietowicz, Z. Sixty seconds on . . . vitamin D. BMJ 371, m3872, doi:10.1136/bmj.m3872 $1205 \quad$ (2020)

120661 Weir, E. K., Thenappan, T., Bhargava, M. \& Chen, Y. Does vitamin D deficiency increase the severity of COVID-19? Clin Med (Lond) 20, e107-e108, doi:10.7861/clinmed.2020-0301

120962 Ritchie, M. E. et al. limma powers differential expression analyses for RNA-sequencing and microarray studies. Nucleic acids research 43, e47, doi:10.1093/nar/gkv007 (2015).

121464 Shannon, P. et al. Cytoscape: a software environment for integrated models of biomolecular interaction networks. Genome Res 13, 2498-2504, doi:10.1101/gr.1239303 (2003). 
121665 Szklarczyk, D. et al. The STRING database in 2017: quality-controlled protein-protein

1217 association networks, made broadly accessible. Nucleic acids research 45, D362-D368,

1218 doi:10.1093/nar/gkw937 (2017).

121966 CoExpNets: Co-expression network management based on WGCNA + k-means. R package 1220 version 0.1.0. v. 0.1 .0 (2019).

122167 Dolgalev, I. msigdbr: MSigDB Gene Sets for Multiple Organisms in a Tidy Data Format. R 1222 package version 7.2.1. . (2020).

1223 
a)

Tripkovic et al. participants, $\mathrm{n}=335$ WE $n=245$, SA $n=90$
Selected 32 subjects from each of the D2 and D3 treatment groups, and 34 from the placebo

For D2 and D3, a range of serum [25OHD] responses to supplementation were chosen

Placebo subjects were chosen at random
Subjects for transcriptome analysis, $\mathrm{n}=98$ WE $n=67$, SA $n=31$

b)

\begin{tabular}{|l|c|c|c|}
\hline Group & $\begin{array}{l}\text { White } \\
\text { Euro }(\mathrm{n})\end{array}$ & $\begin{array}{l}\text { South } \\
\text { Asian }(\mathrm{n})\end{array}$ & Total $(\mathrm{n})$ \\
\hline D2 & 21 & 11 & 32 \\
\hline D3 & 21 & 11 & 32 \\
\hline Placebo & 25 & 8 & 33 \\
\hline
\end{tabular}

c)

25(OH)D2

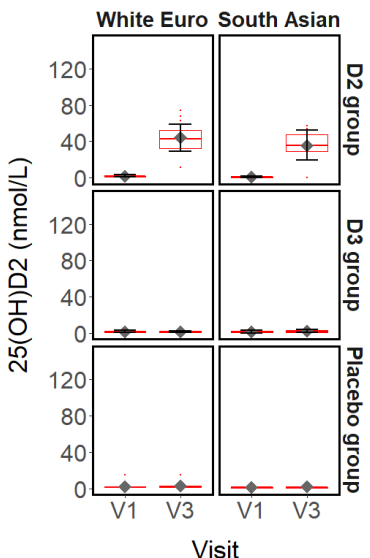

25(OH)D3

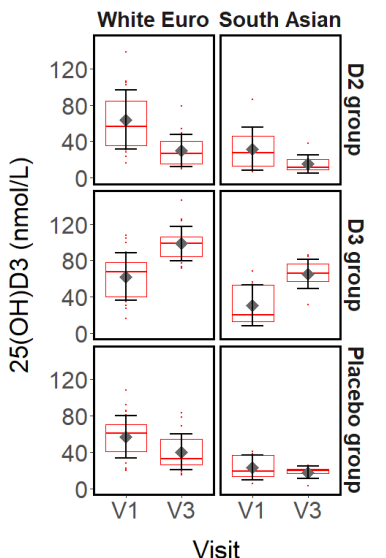

Total 25(OH)D

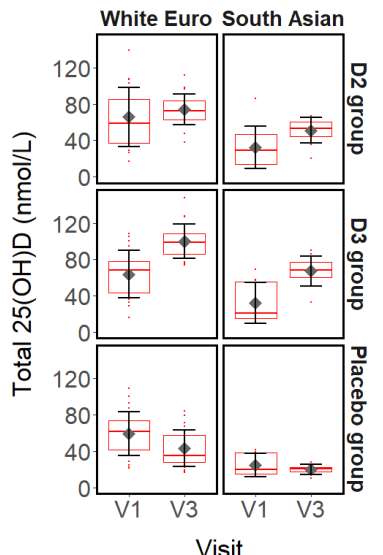

PTH

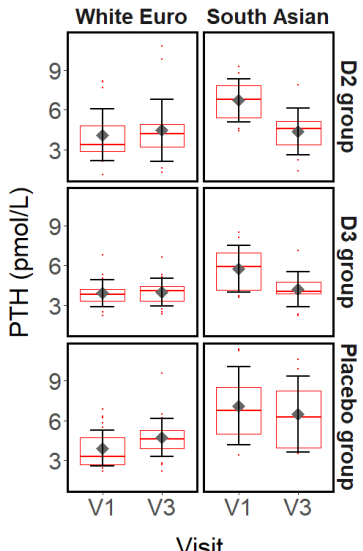

Adj. calcium

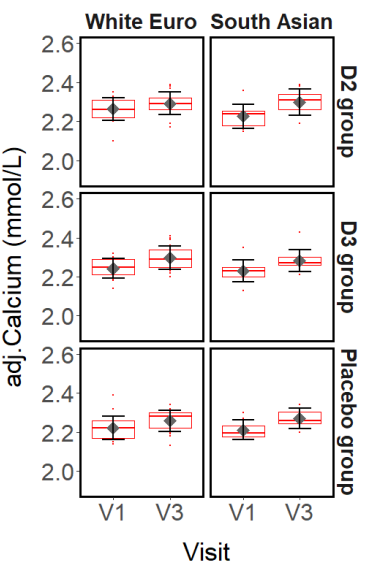

Fig. 1. 
a)

\begin{tabular}{|c|c|c|}
\hline Comparison & $\begin{array}{l}\text { Sig. down } \\
\text { probes (genes) }\end{array}$ & $\begin{array}{l}\text { Sig. up } \\
\text { probes (genes) }\end{array}$ \\
\hline WE D2 V3 v V1 & $523(498)$ & $369(350)$ \\
\hline WE D3 V3 v V1 & $997(943)$ & $342(329)$ \\
\hline WE P V3 ₹ V1 & $1171(1096)$ & $605(565)$ \\
\hline SA D2 V3 v V1 & $1(1)$ & $0(0)$ \\
\hline SA D3 V3 v V1 & $1(1)$ & $0(0)$ \\
\hline SA P V3 v V1 & $191(189)$ & $436(408)$ \\
\hline [WE D2 V3 v V1] [WE P V3 v V1] & $0(0)$ & $0(0)$ \\
\hline [WE D3 V3 V1] v [WE P V3 V1] & $0(0)$ & $0(0)$ \\
\hline 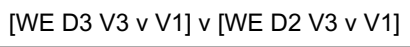 & $0(0)$ & $0(0)$ \\
\hline 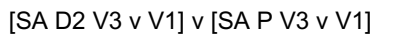 & $0(0)$ & $0(0)$ \\
\hline 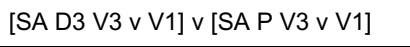 & $2(2)$ & $3(3)$ \\
\hline 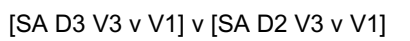 & $0(0)$ & $0(0)$ \\
\hline
\end{tabular}

c)

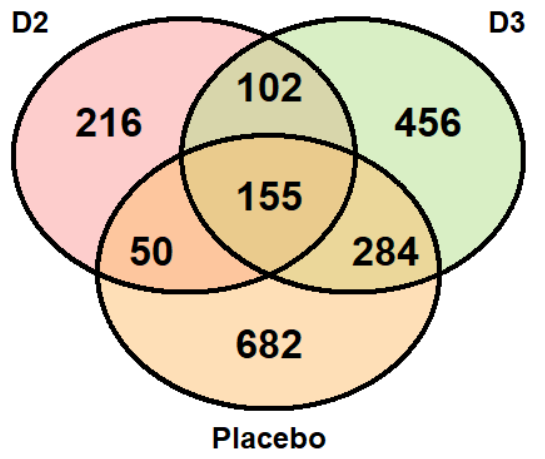

Down, WE V3 v V1

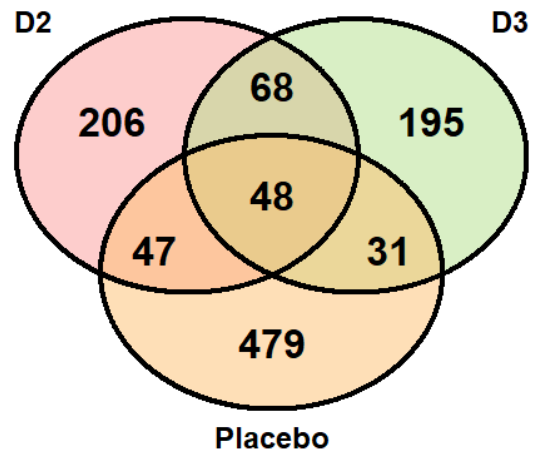

Up, WE V3 v V1 b)

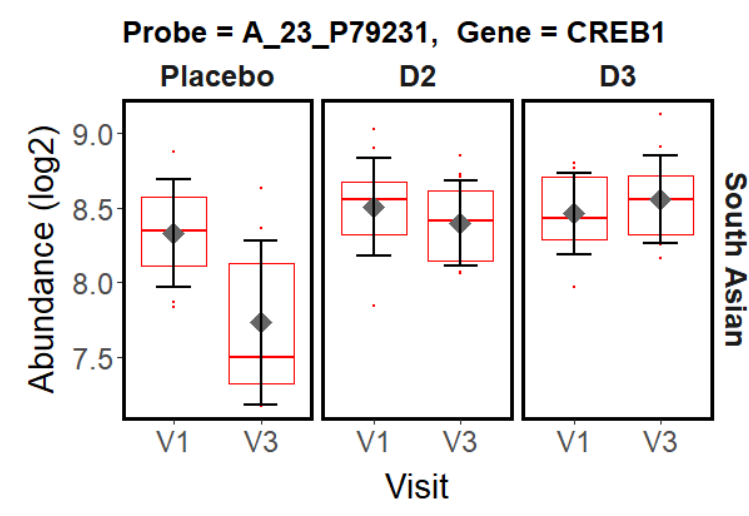

d)

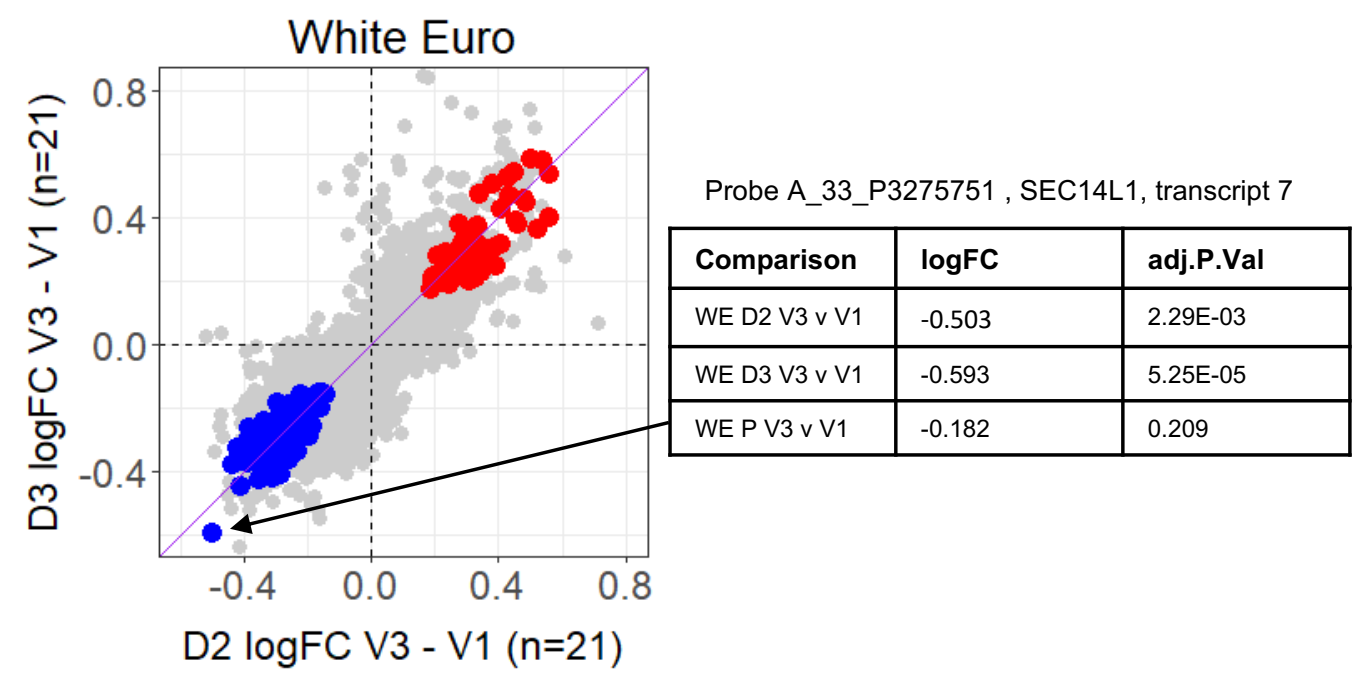

Fig. 2. 
a) Significantly down-regulated by $D_{2}$ and $D_{3}$, but not placebo (blue points in Fig. 2d)

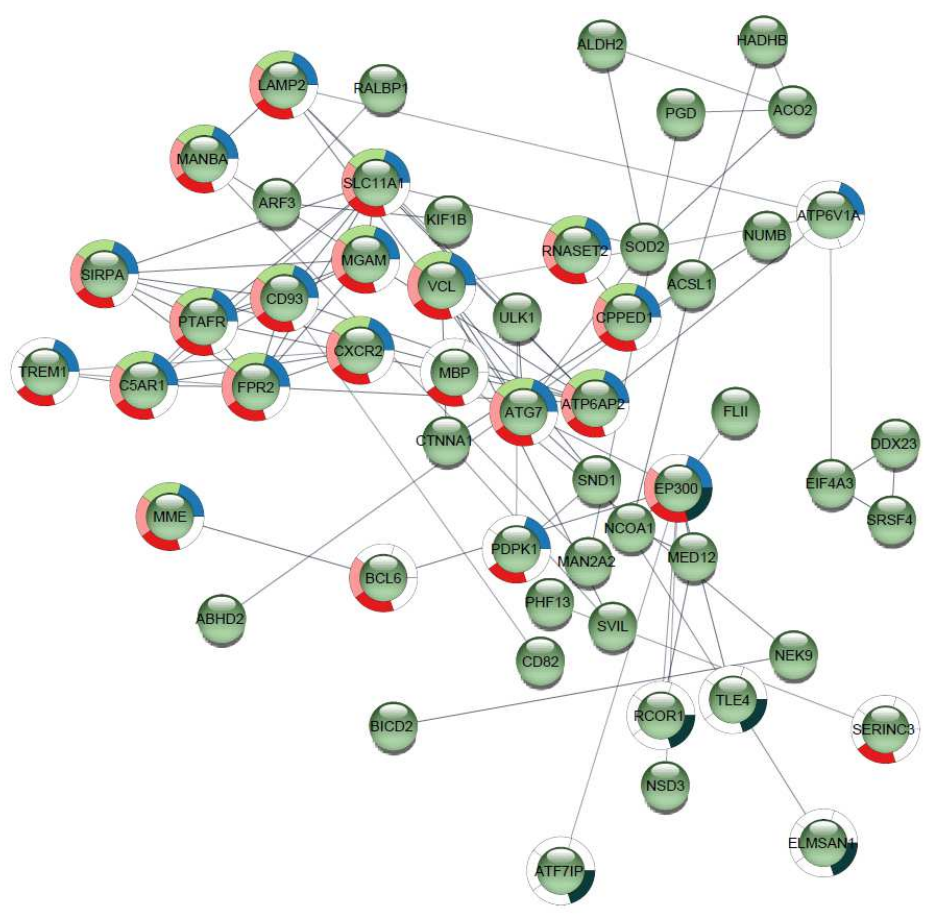

$\underline{\text { Significant functional enrichment (FDR }<0.05)}$

Immune system process

Innate immune system

Transcription factor complex

Neutrophil degranulation

Leukocyte activation b) Significantly up-regulated by $D_{2}$ and $D_{3}$, but not placebo (red points in Fig. 2d)

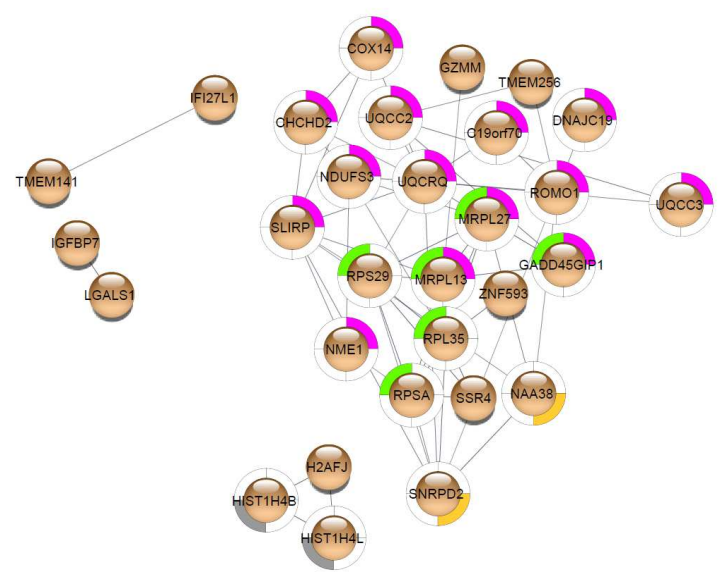

$\underline{\text { Significant functional enrichment }(F D R<0.05}$

Mitochondrion

Ribosomal protein

snRNP Sm proteins

Histone $\mathrm{H} 4$

Fig. 3. 
a)

Significant GO BP functional categories associated with down-regulated probes in the D2 or D3 treatment groups, but not the placebo

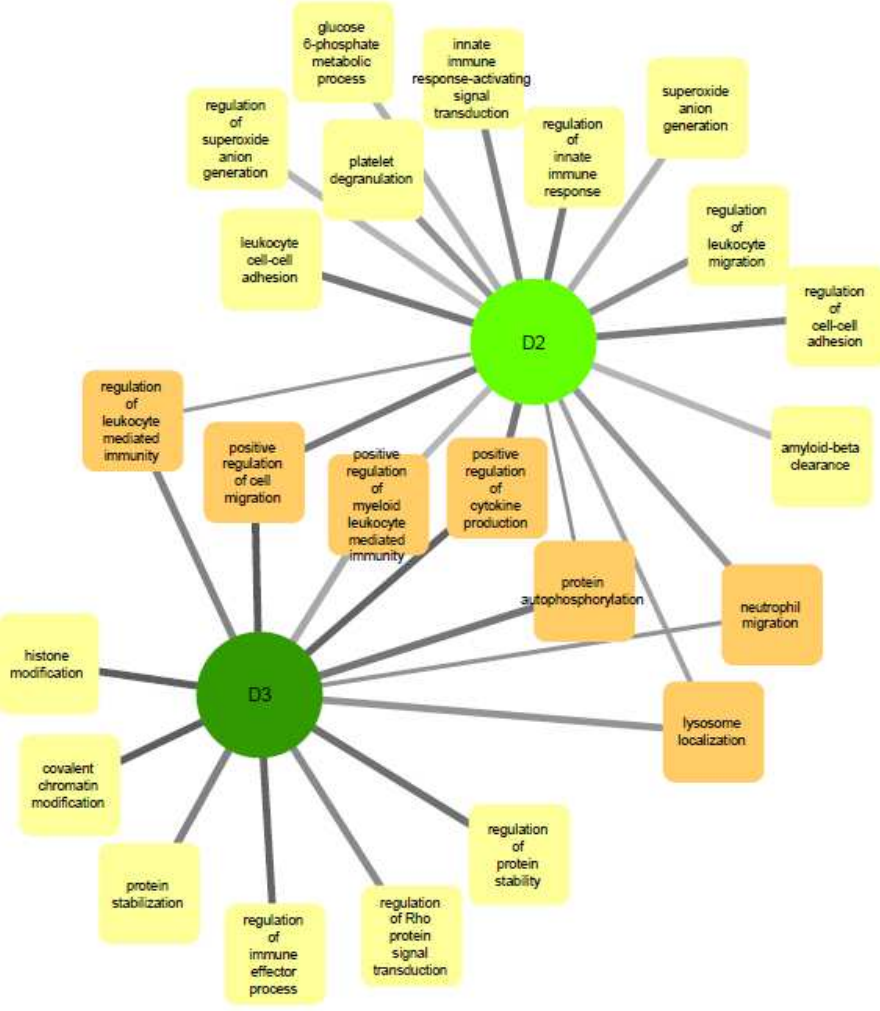

b) Significant Reactome PA categories associated with down-regulated probes in the D2 or D3 treatment groups, but not the placebo

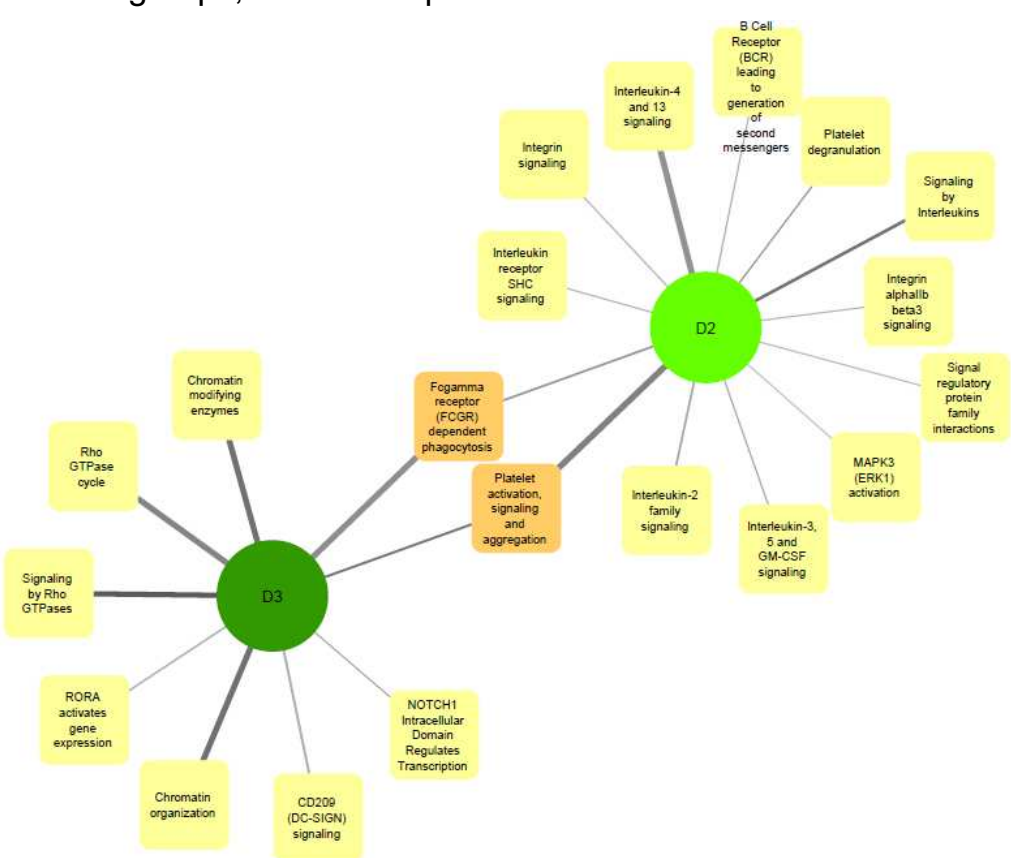

Fig. 4. 
a) Significant GO CC functional categories associated with up-regulated probes in the D2 or D3 treatment groups, but not the placebo

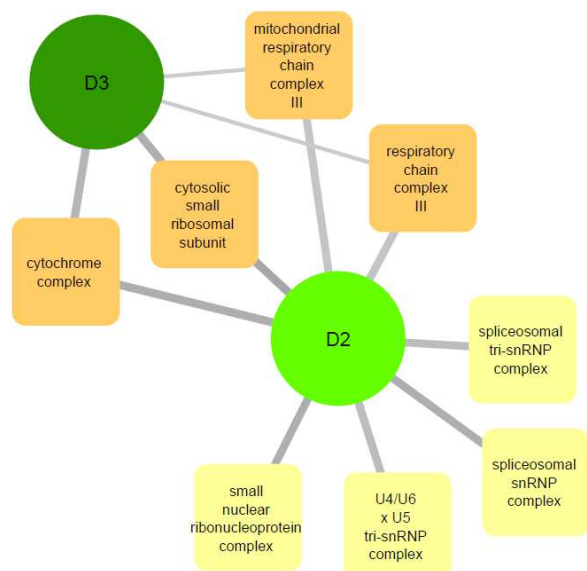

b) Significant GO BP functional categories associated with up-regulated probes in the D2 or D3 treatment groups, but not the placebo

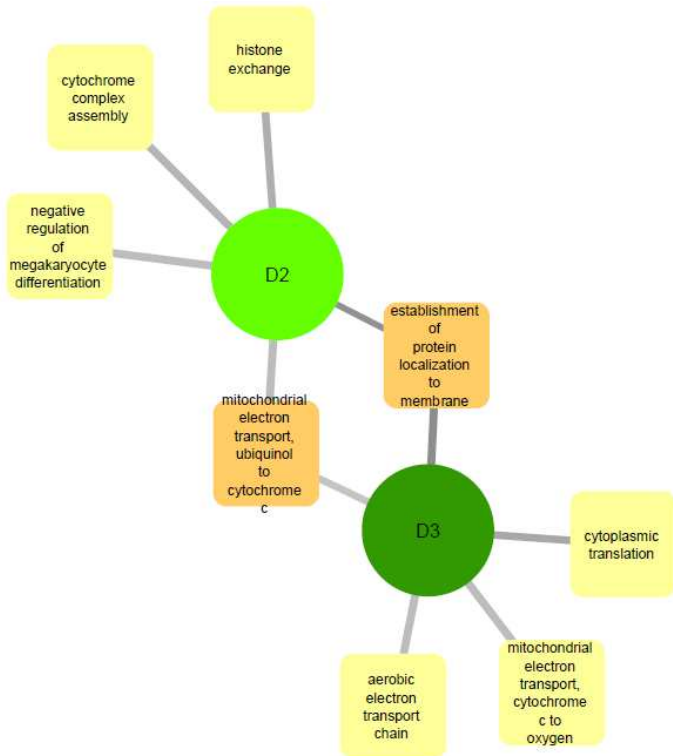

Fig. 5. 
a) Co-expression modules from WE subject data $(n=67,134$ microarrays)

\section{$\underline{\text { GO enrichment }}$}

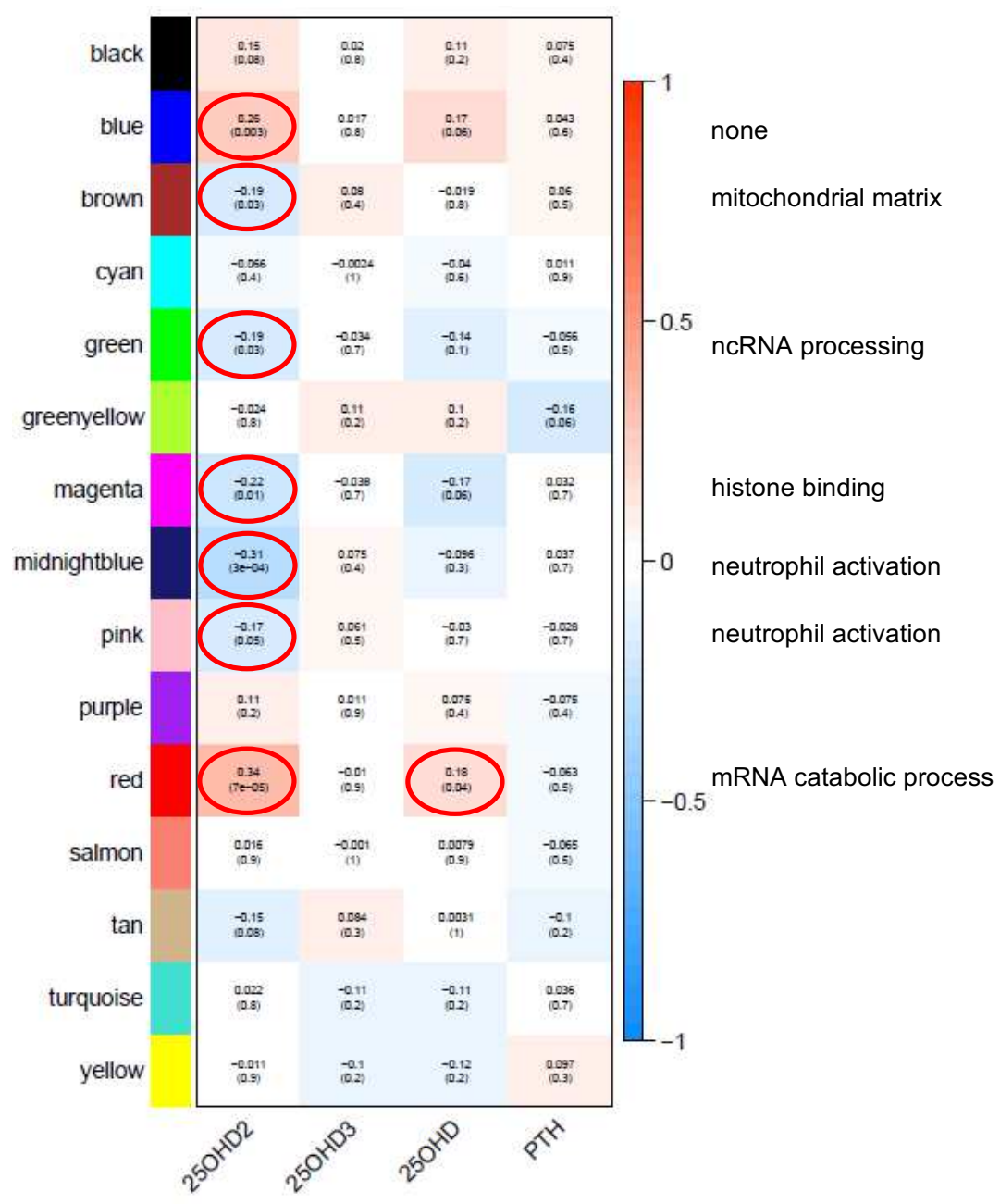
( $n=30,60$ microarrays)

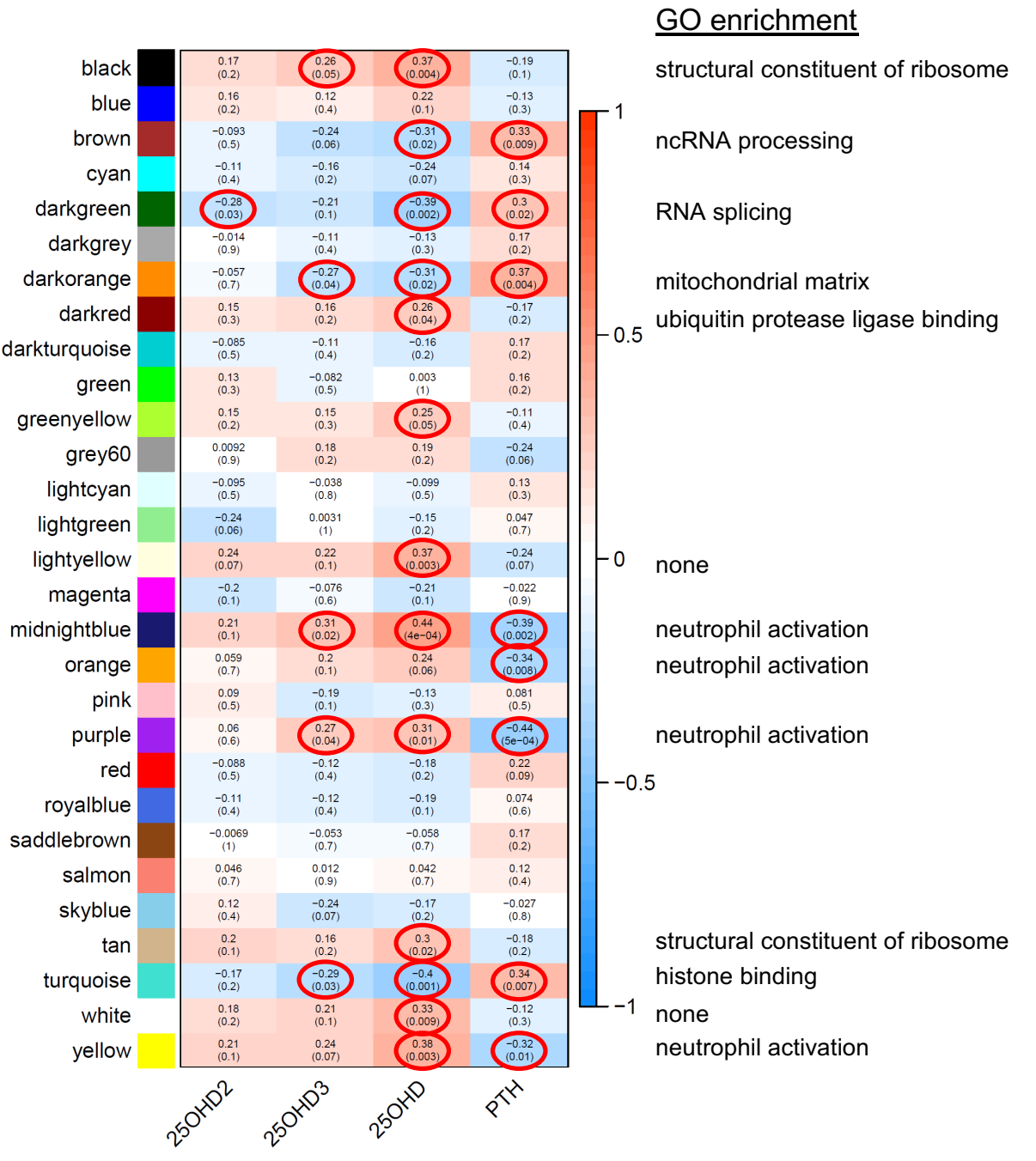

Fig. 6. 


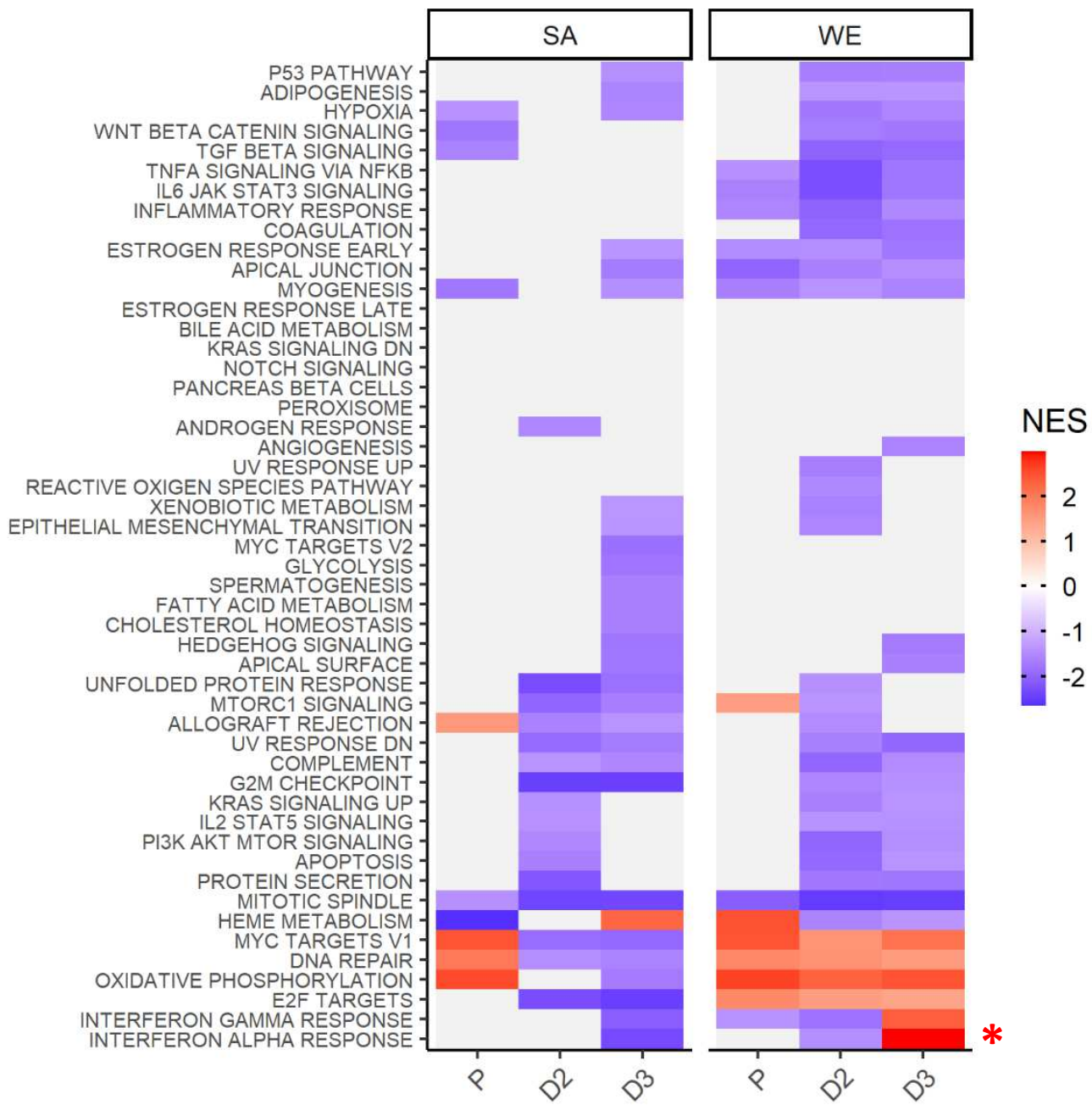

Fig. 7. 
a)

\begin{tabular}{|c|c|c|c|c|c|}
\hline Probe & Systematic Name & Symbol & Gene & $\log \mathrm{FC}$ & adj.P.Val \\
\hline A_23_P79231 & NM_134442 & CREB1 & CAMP responsive element binding protein 1 & 0.698193 & 0.021322 \\
\hline A_21_P0014443 & THC2548652 & & NA & 0.676465 & 0.001531 \\
\hline A_33_P3315320 & NM_173478 & CNTD1 & cyclin N-terminal domain containing 1 & 0.589848 & 0.007448 \\
\hline A_23_P218706 & NM_024325 & ZNF343 & zinc finger protein 343 & -0.46626 & 0.025991 \\
\hline A_33_P3275998 & NM_004703 & RABEP1 & rabaptin, RAB GTPase binding effector protein 1 & -0.43299 & 0.049483 \\
\hline
\end{tabular}

b)
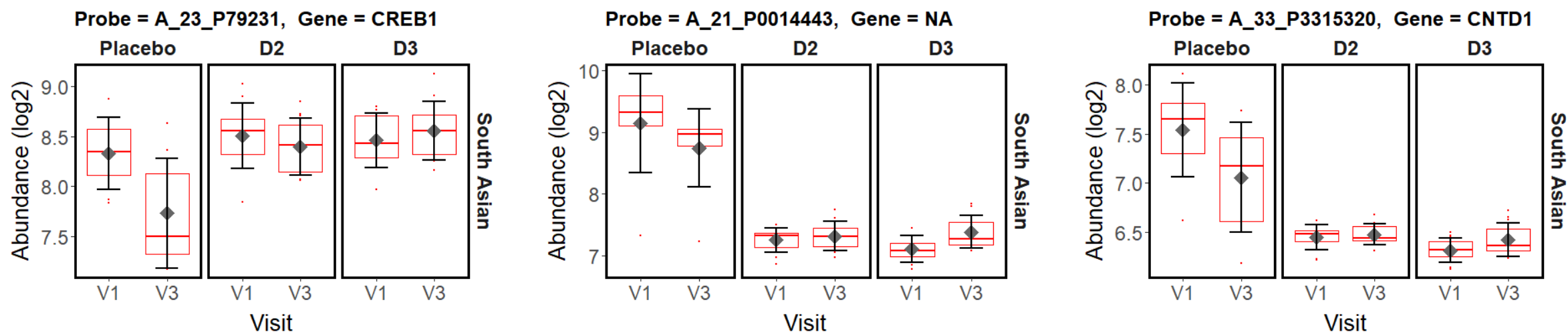

Probe $=$ A_23_P218706, Gene $=$ ZNF343
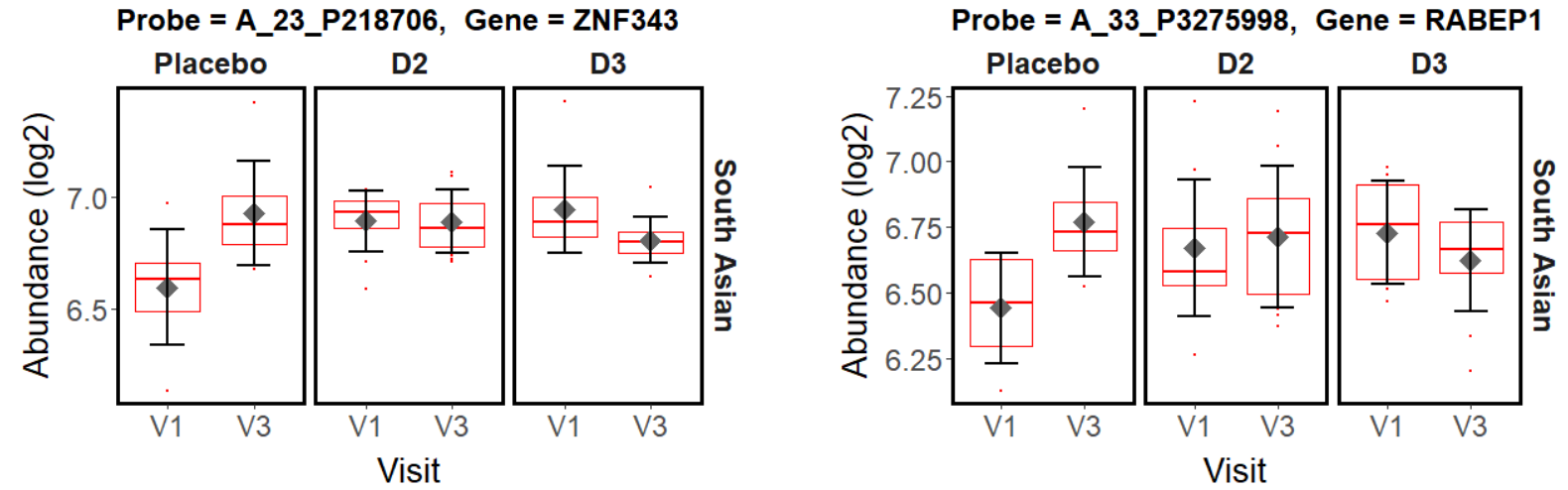

Supplementary Fig. 1. Summary of the probes identified as being significantly different in the difference in difference analysis of the SA cohort (comparison [SA D3 V3 v V1] v [SA P V3 v V1] in Fig. 2a). A) Differential expression test results. B) Probe signal abundance $(\log 2)$ distribution across the experimental treatments in the SA cohort. 
a)

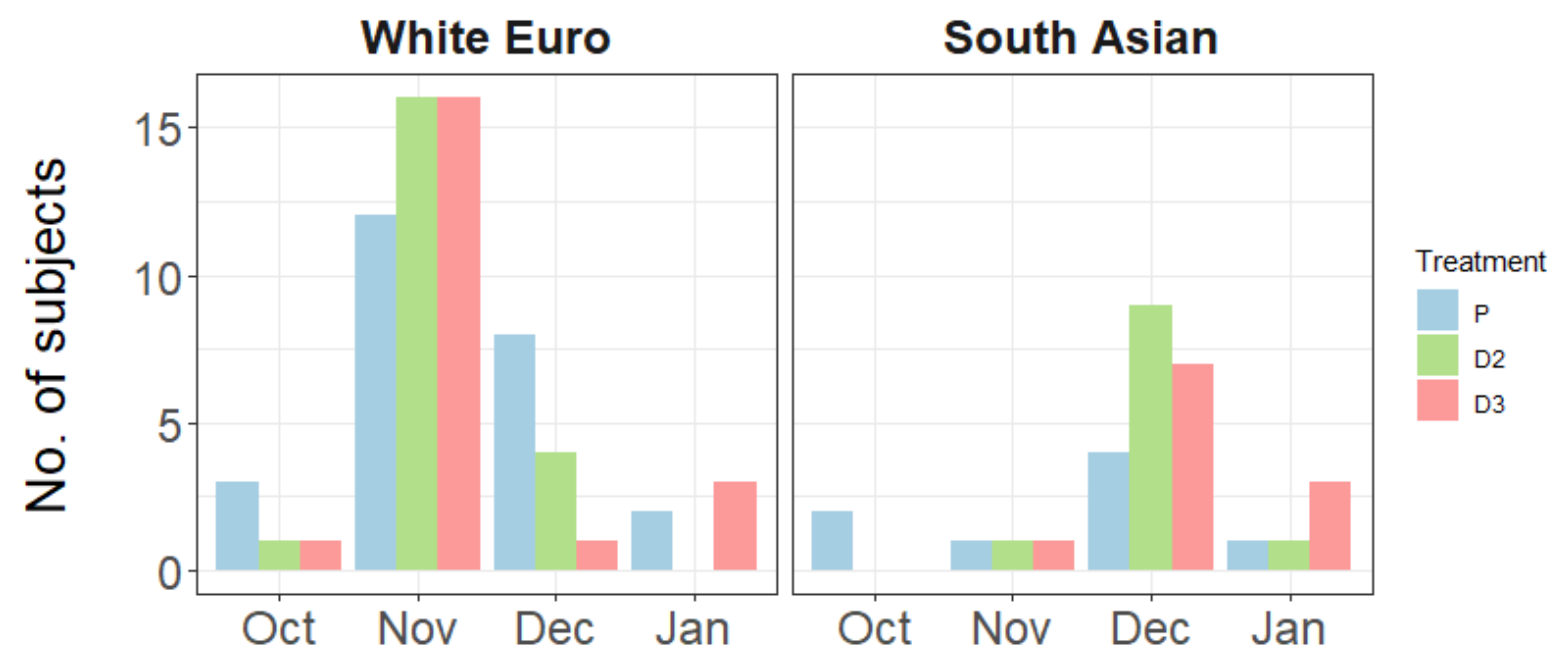

\section{Month of visit 1}

b)

\begin{tabular}{|l|c|c|c|}
\hline & \multicolumn{3}{|c|}{ Enrichment p-value (Fisher's exact test) using the significant gene sets below } \\
\hline Comparison & Down V3 v V1 & Up V3 v V1 & All V3 v V1 \\
\hline WE P & $1.97 \mathrm{E}-29$ & $2.49 \mathrm{E}-07$ & $8.69 \mathrm{E}-35$ \\
\hline WE D2 & $4.95 \mathrm{E}-28$ & $1.14 \mathrm{E}-01$ & $4.69 \mathrm{E}-22$ \\
\hline WE D3 & $8.80 \mathrm{E}-35$ & $7.77 \mathrm{E}-01$ & $5.49 \mathrm{E}-27$ \\
\hline
\end{tabular}

Supplementary Fig. 2. Seasonal gene expression as a feature of the 12-week study $\left(P=\right.$ placebo, $D 2=$ vitamin $D_{2}, D 3=$ vitamin $\left.D_{3}\right)$. a) Summary of the timing of the V1 samples taken in the study (for each individual, V3 samples were taken 12 weeks after the V1 sample). b) Significant over-representation of the seasonal genes identified by Dopico et al (2015) Nat Commun, 6, 7000 (BABYDIET dataset) in the lists of genes identified as significantly changing in the white European (WE) placebo, $D_{2}$ and $D_{3}$ treatment groups. The fisher.test function in $\mathrm{R}$ was used to examine the significance of the association (contingency) between classification as a seasonally expressed gene and classification as a gene significantly differently expressed in each of the nine groups shown in the table. The gene 'universe' was defined as all genes in the genome annotated with an ENSEMBL gene identifier. 


\section{PCA analysis of the 97 participants - V3 vs V1}

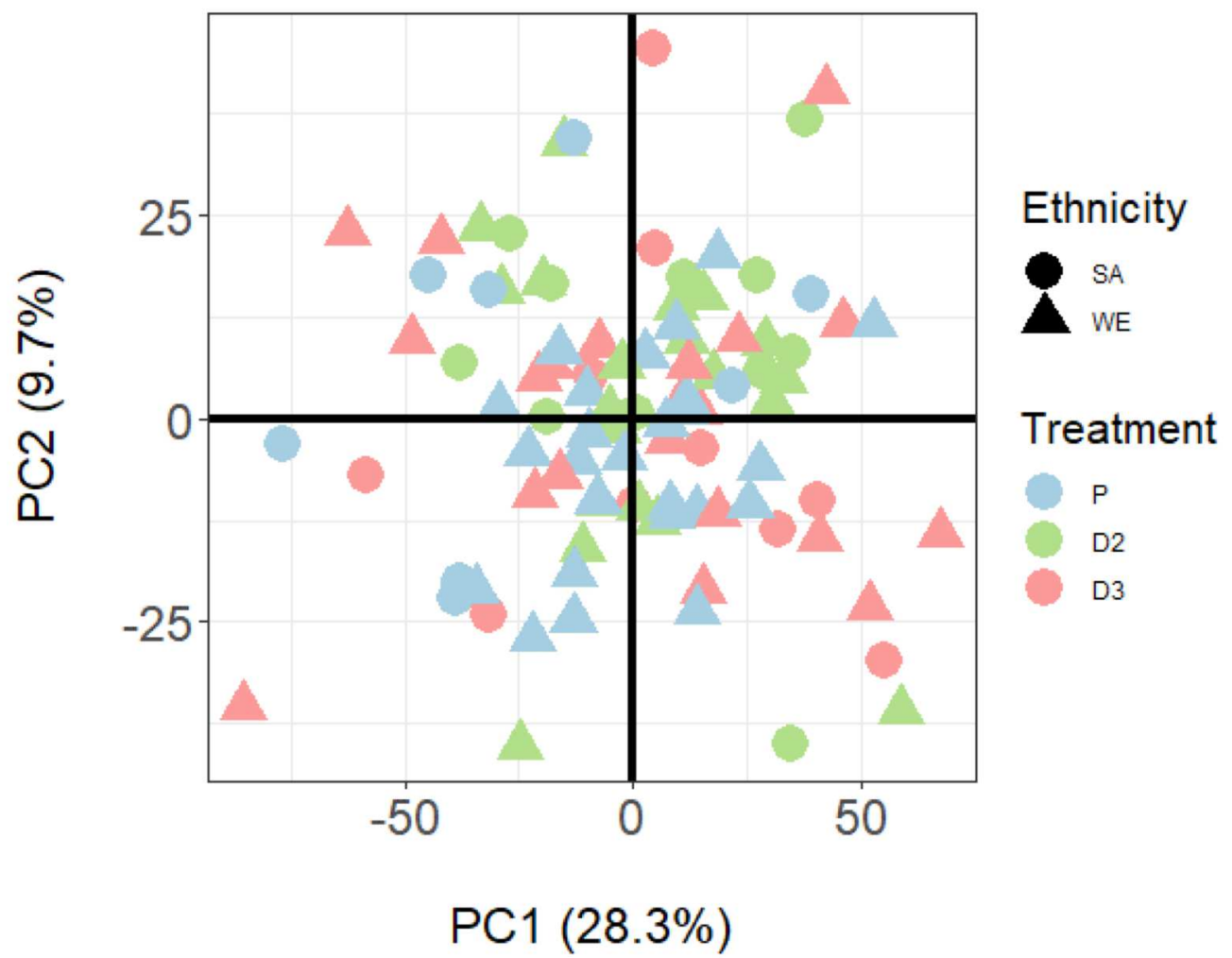

\section{Supplementary Fig. 3.}

Principal component analysis of the transcriptome data for the 97 subjects passing quality control. For each subject participant, a value defined as $\mathrm{V} 3-\mathrm{V} 1$ was calculated for all the microarray gene probes in the normalised data by subtracting the abundance of each probe signal in the $\mathrm{V} 1$ sample from the abundance of the same probe signal in the $\mathrm{V} 3$ sample. The resulting matrix of values was analysed using the pca function in the R package pcaMethods (Stacklies, W., Redestig, H., Scholz, M., Walther, D. and Selbig, J. Bioinformatics, 2007, 23, 1164-1167). No obvious clustering by treatment group and/or ethnicity is observed. 
Figures

a)

Tripkovic et al. participants, $\mathrm{n}=335$ WE $n=245, S A n=90$

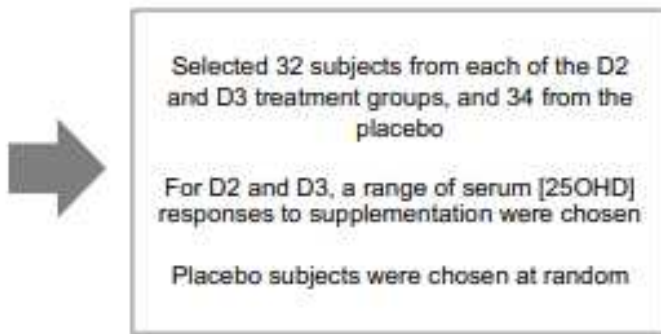

b)

\begin{tabular}{|l|c|c|c|}
\hline Group & $\begin{array}{l}\text { White } \\
\text { Euro }(\mathrm{n})\end{array}$ & $\begin{array}{l}\text { South } \\
\text { Asian }(\mathrm{n})\end{array}$ & Total (n) \\
\hline D2 & 21 & 11 & 32 \\
\hline D3 & 21 & 11 & 32 \\
\hline Placebo & 25 & 8 & 33 \\
\hline
\end{tabular}

c)
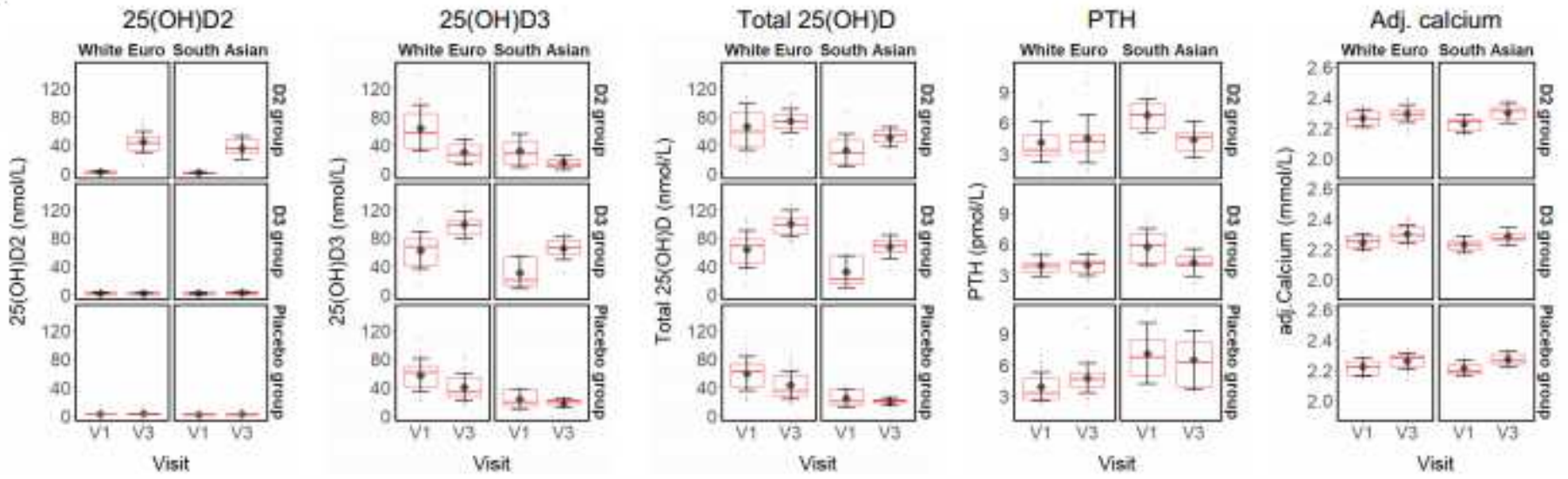

Figure 1

Please see the manuscript file to view the figure caption. 
a)

\begin{tabular}{|c|c|c|}
\hline Comparison & $\begin{array}{l}\text { Sig. down } \\
\text { probes (genes) }\end{array}$ & $\begin{array}{l}\text { Sig. up } \\
\text { probes (genes) }\end{array}$ \\
\hline WE $D 2$ V3 V V1 & 523 (499) & $369(350)$ \\
\hline WE D3V3 V V1 & 997 (943) & 342 (329) \\
\hline WEPV $3 \times V 1$ & 1171 (1096) & $605(565)$ \\
\hline SAD2 V3 v V1 & $1(1)$ & $O(0)$ \\
\hline SA D3 V3 V V1 & $1(1)$ & $0(0)$ \\
\hline SAPV $3 \vee V 1$ & 191 (189) & $436(408)$ \\
\hline 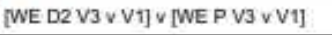 & $0(0)$ & $0(0)$ \\
\hline$[W E$ D3 V3 $\vee$ V1] $\vee[W E P$ V 3 $\vee$ V1] & $0(0)$ & $0(0)$ \\
\hline [WE D3 V3 V V1] $\vee[W E$ D2 V3 v V1] & $0(0)$ & $0(0)$ \\
\hline [SA D2 V3 v V1] v [SAP V3 v V1] & $0(0)$ & $0(0)$ \\
\hline [SA D3 V3 V V1] V [SAP V3 V V1] & $2(2)$ & $3(3)$ \\
\hline [SA D3 V3 v V1] v [SA D2 V3 v V1] & $O(0)$ & $0(0)$ \\
\hline
\end{tabular}

c)

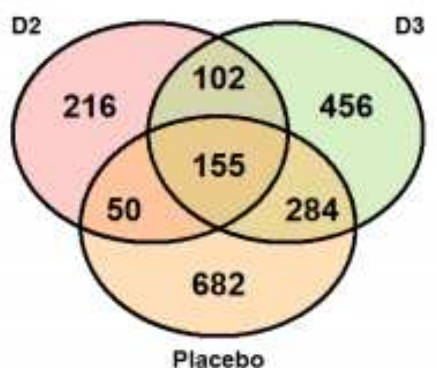

Down, WE V3 v V1

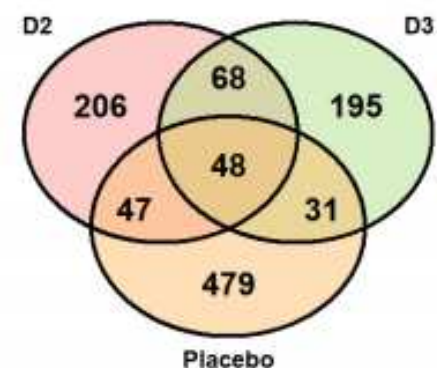

Up, WE V3 v V1 b)

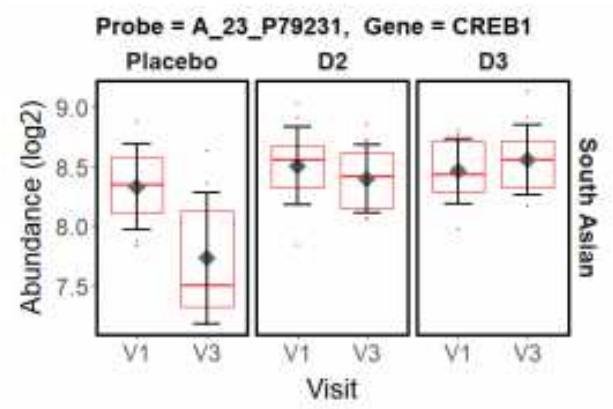

d)

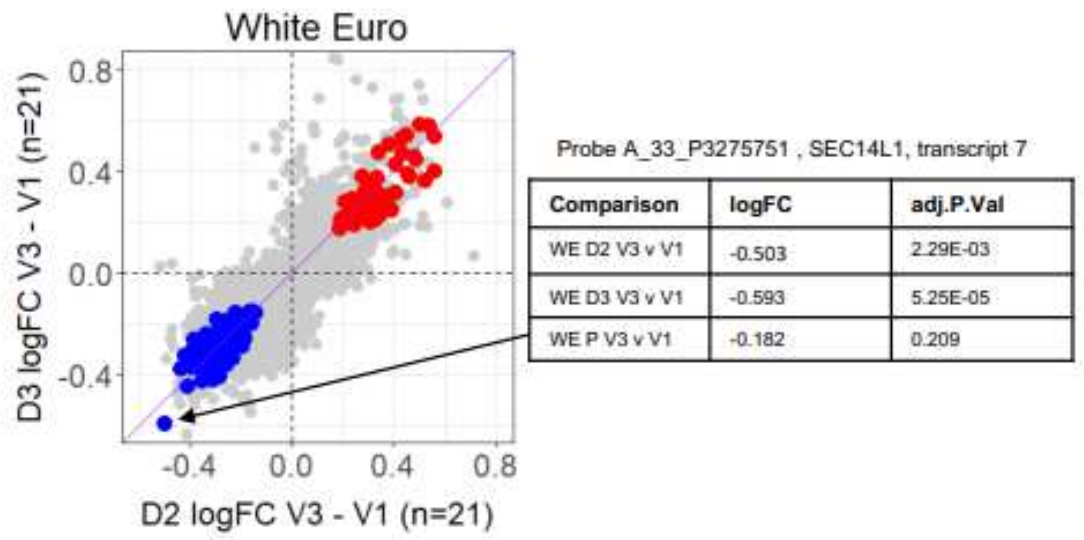

\section{Figure 2}

Please see the manuscript file to view the figure caption. 
a) Significantly down-regulated by $D_{2}$ and $D_{3}$, but not placebo (blue points in Fig. 2d)

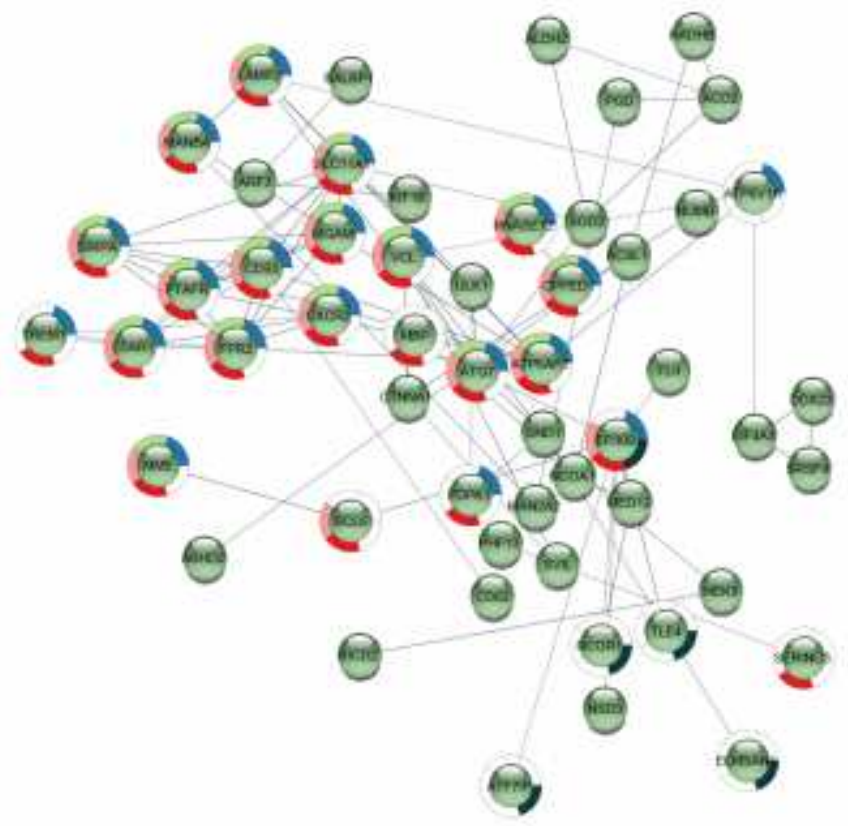

Significant functional enrichment (FDR<0.05)

immune system process

innate immune system

Transcription factor complex

Neutraphil degranulation

Leukocyte activation

\section{Figure 3}

Please see the manuscript file to view the figure caption. b) Significantly up-regulated by $D_{2}$ and $D_{3}$, but not placebo (red points in Fig. 2d)

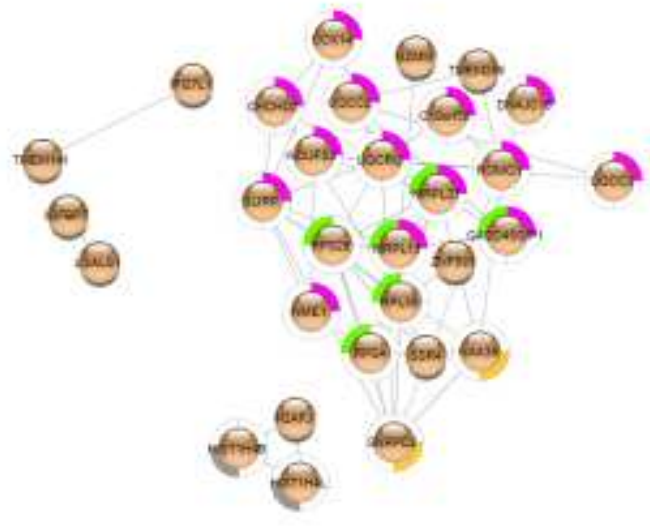

Significant functional enrichment (FDR $<0.05$ )

Mitochondrion

Pibasomal protein

snRNP Sm proteins

Histone $\mathrm{H}_{4}$ 
a)

Significant GO BP functional categories associated with down-regulated probes in the D2 or D3 treatment groups, but not the placebo

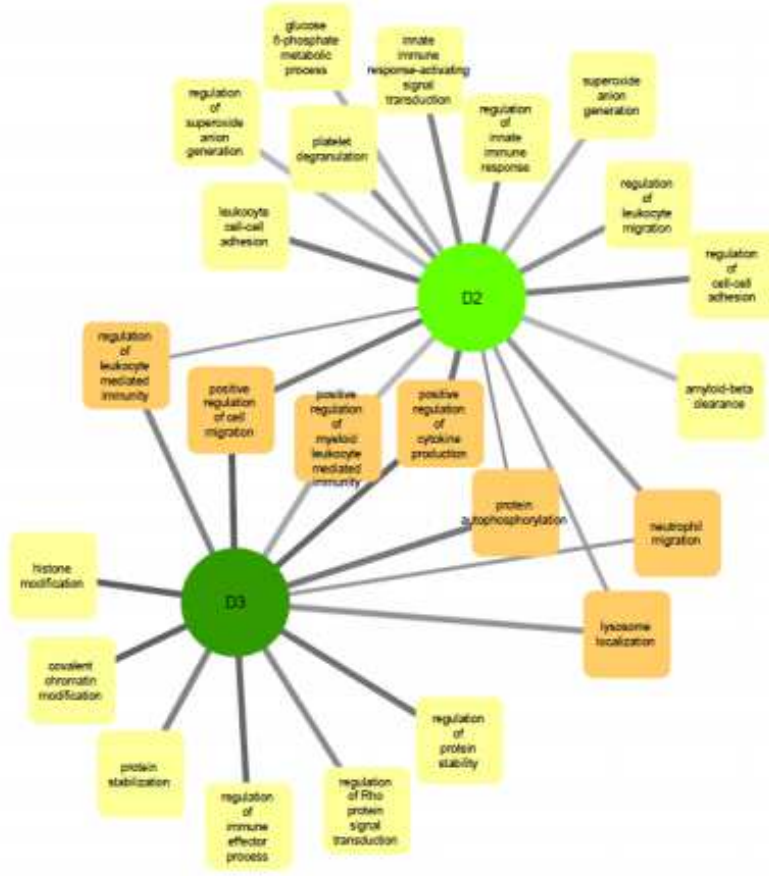

b) Significant Reactome PA categories associated with down-regulated probes in the D2 or D3 treatment groups, but not the placebo

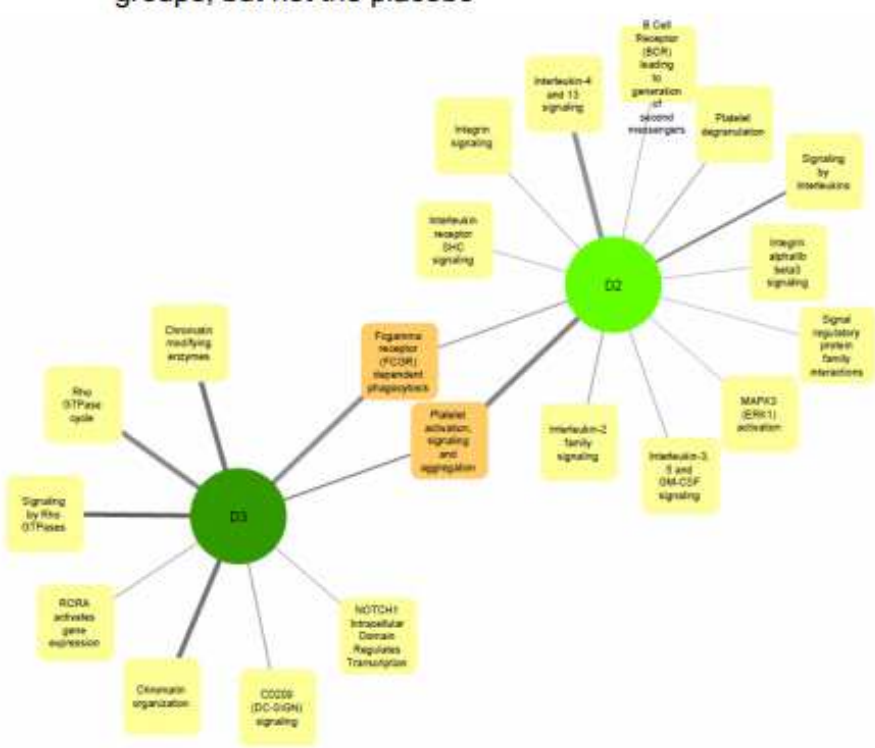

\section{Figure 4}

Please see the manuscript file to view the figure caption.

a)

Significant GO CC functional categories associated with up-regulated probes in the D2 or D3 treatment groups, but not the placebo

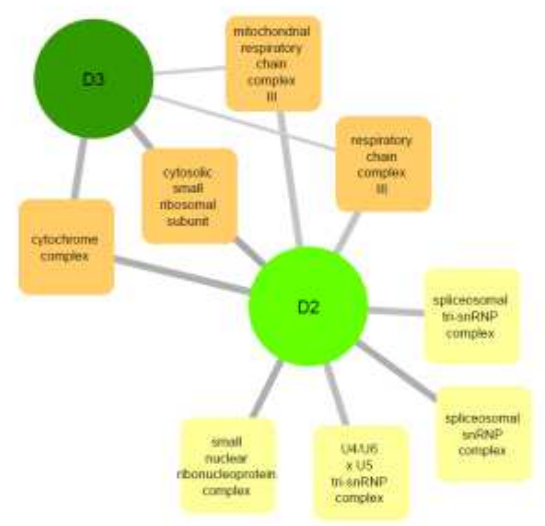

b) Significant GO BP functional categories associated with up-regulated probes in the D2 or D3 treatment groups, but not the placebo

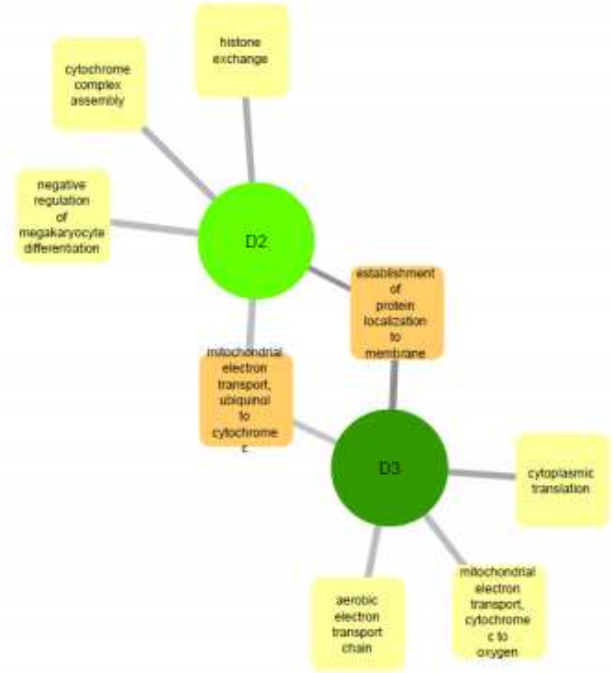

Figure 5

Please see the manuscript file to view the figure caption. 
a) Co-expression modules from WE subject data ( $n=67,134$ microarrays)

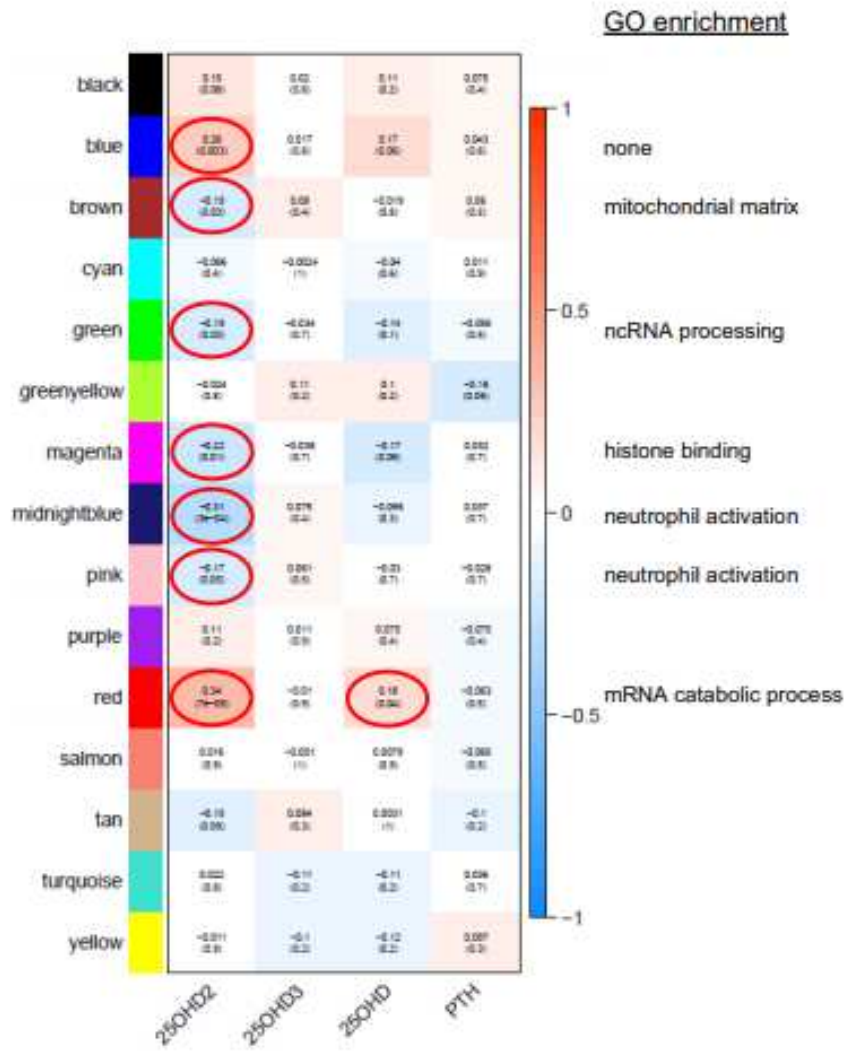

b) Co-expression modules from SA subject data ( $n=30,60$ microarrays)

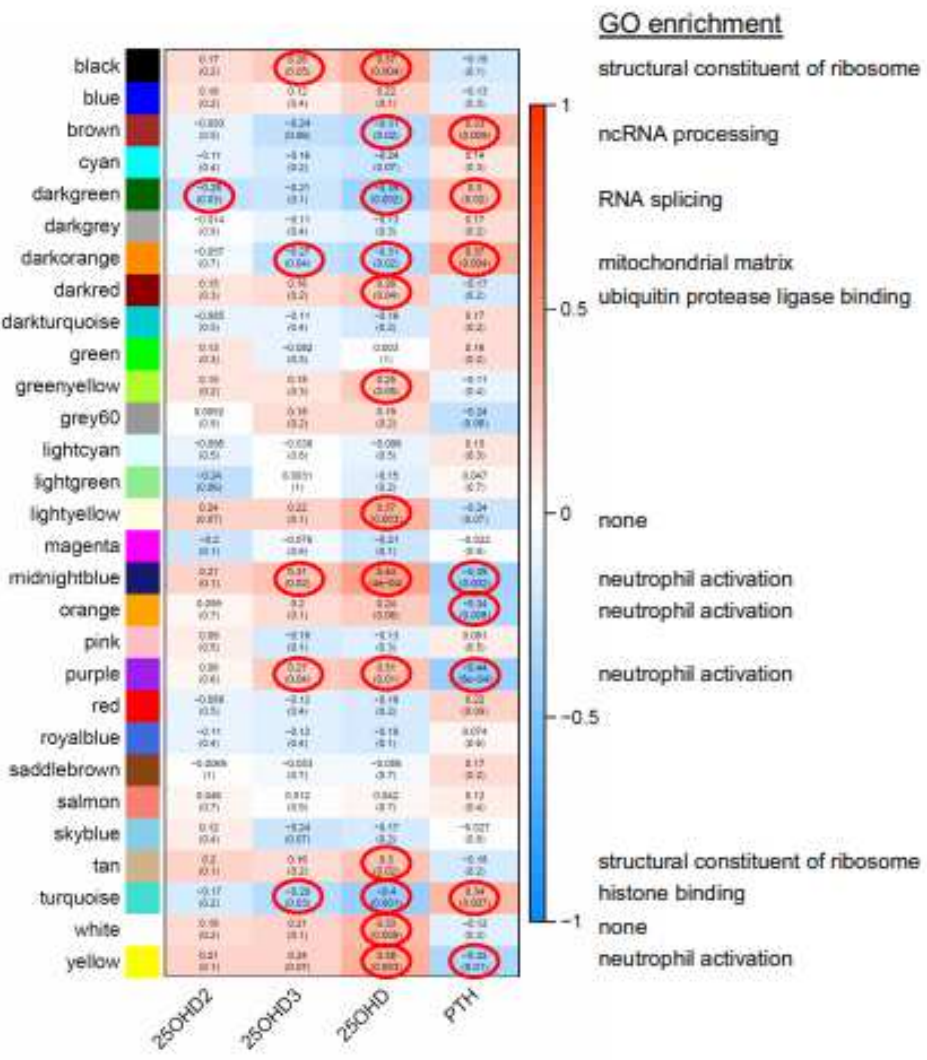

\section{Figure 6}

Please see the manuscript file to view the figure caption. 


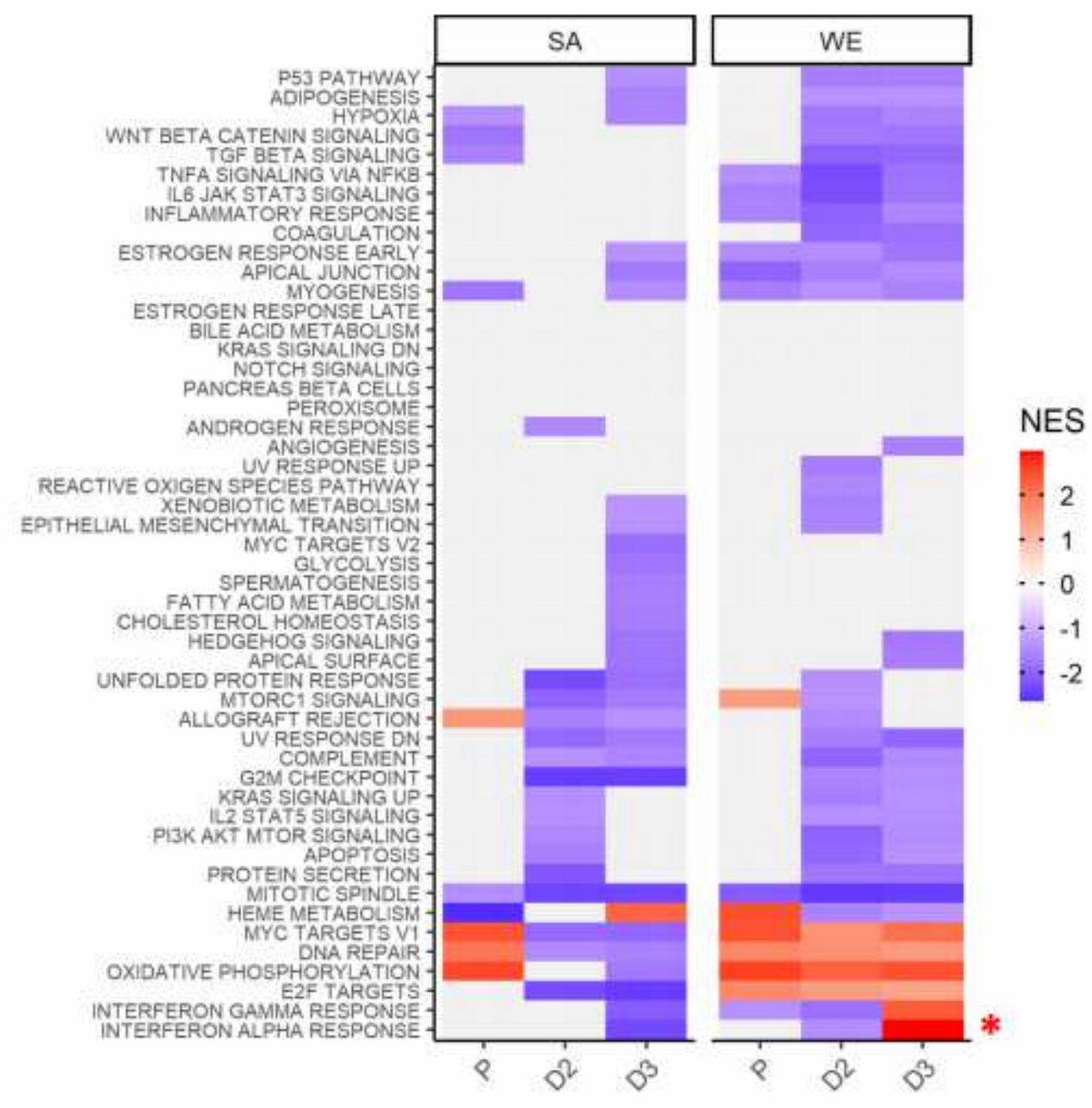

Figure 7

Please see the manuscript file to view the figure caption.

\section{Supplementary Files}

This is a list of supplementary files associated with this preprint. Click to download.

- Supplementarydatafile1 anonymised.xlsx

- Supplementarydatafile2.xlsx

- Supplementarydatafile3.xlsx

- Supplementarydatafile4.xlsx

- Supplementarydatafile5.pdf

- Supplementarydatafile6.xlsx

- Supplementarydatafile7.xlsx

- Supplementarydatafile8.xlsx 
- SupplementaryDataFile9.xIsx 